\title{
FFG Wirkungsmonitoring 2013
}

(der im Jahr 2009 abgeschlossenen Projekte) 
Diese Studie wurde im Auftrag der Österreichischen Forschungsförderungsgesellschaft (FFG) durchgeführt.

\section{VERFASSER DES BERICHTS:}

Peter Kaufmann

Laurenz Wolf

INTERNES REVIEW:

Sonja Sheikh

LAYOUT:

Martina Gugerell

Die vorliegende Studie wurde nach allen Maßstäben der Sorgfalt erstellt.

Die KMU Forschung Austria übernimmt jedoch keine Haftung für Schäden oder Folgeschäden, die auf diese Studie oder auf mögliche fehlerhafte Angaben zurückgehen.

Dieses Werk ist urheberrechtlich geschützt. Jede Art von Nachdruck, Vervielfältigung, Verbreitung, Wiedergabe, Übersetzung oder Einspeicherung und Verwendung in Datenverarbeitungssystemen, und sei es auch nur auszugsweise, ist nur mit ausdrücklicher Zustimmung der KMU Forschung Austria gestattet.

Mitglied bei:

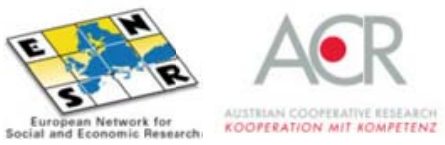




\section{Inhaltsverzeichnis}

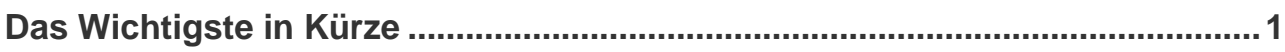

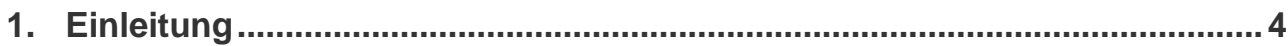

2. Methodik und Eckdaten der analysierten Unternehmen..............................5

3. Positionierung der Projekte im Portfolio der Unternehmen ........................13

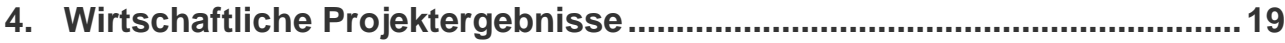

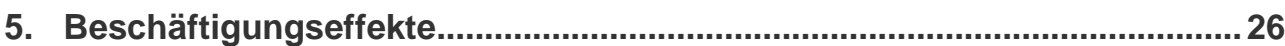

6. Auswirkungen auf die Forschungs-, Entwicklungs-, und Innovationsaktivitäten der Unternehmen ......................................................... 35

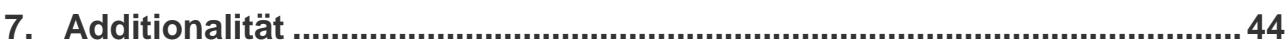

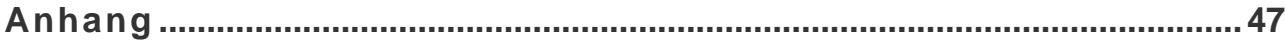

Berechnungsformeln

Tabellen 


\section{Tabellenverzeichnis}

Tabelle 1 Verteilung der in die Analyse einbezogenen, sowie 2009 abgeschlossenen Projekte nach Programmen.....

Tabelle 2 Verteilung der in die Analyse einbezogenen, sowie in den Jahren 2007-2009 abgeschlossenen Projekte, Anzahl

Tabelle 3 Zuordnung der Projekte zur neuen FFG Klassifikation der Förderinstrumente

Tabelle 4 Verteilung der 2009 abgeschlossenen Projekte nach Beschäftigtengrößenklassen der Unternehmen

Tabelle 5 Verteilung der analysierten und 2009 abgeschlossenen Projekte nach ÖNACE-Klassifikation der Projekte

Tabelle 6 Verteilung der Projekte nach der Rolle des Unternehmens innerhalb des Gesamt-Projektes

Tabelle 7 Verteilung der 2009 abgeschlossenen Projekte nach der Rolle des Projekts im Portfolio des Unternehmens

Tabelle 8 Verteilung der 2009 abgeschlossenen Projekte nach der Rolle des Projekts hinsichtlich der FuE Aktivitäten im Unternehmen

Tabelle 9 Erreichung des Projektzieles aus technischer bzw. wirtschaftlicher Sicht nach Basisprogramm und sonstigen Programmen, 2009 abgeschlossene Projekte.

Tabelle 10 Erreichung des Projektzieles aus technischer und wirtschaftlicher Sicht nach Unternehmensgrößenklassen, 2009 abgeschlossene Projekte.

Tabelle 11 Wirtschaftliche Verwertung der Projektergebnisse der im Jahr 2009 abgeschlossenen Projekte und vergebene Fördermittel

Tabelle 12 Direkte wirtschaftliche Ergebnisse der Befragungsteilnehmer der im Jahr 2009 abgeschlossenen Projekte, in Mio $€$

Tabelle 13 Fördermultiplikatoren

Tabelle 14 Barwerte der Fördermittel je gesicherten bzw. geschaffenen Arbeitsplatz durch die im Jahr 2009 abgeschlossenen Projekte, nach Größenklassen in $€$.

Tabelle 15 Durchschnittlicher Barwert der Fördermittel je gesicherten bzw. geschaffenen Arbeitsplatz der in den Jahren 2005 bis 2009 abgeschlossenen Projekte, nach Größenklassen

Tabelle 16 Arten der Innovation von im Jahr 2009 abgeschlossenen Projekten . 36

Tabelle 17 Auswirkungen der 2009 abgeschlossenen Projekte auf unterschiedliche Bereiche im Unternehmen, in Prozent

Tabelle 18 Auswirkungen auf Kooperationen und Märkte, 2009 abgeschlossen 40

Tabelle 19 Kontakte nach Bereichen, 2009 abgeschlossen ............................... 41

Tabelle 20 KMU-Definition der Europäische Kommission

Tabelle 21 Frage 8: Wurde das Projektziel aus technisch / wirtschaftlicher Sicht erreicht? Im Bundesländervergleich 
Tabelle 22 Frage 8: Wurde das Projektziel aus technisch/wirtschaftlicher Sicht erreicht? Nach Beschäftigtengrößenklassen..

Tabelle 23 Frage 8: Wurde das Projektziel aus technisch/wirtschaftlicher Sicht erreicht? Nach ÖNACE Klassifikation der Projekte.

Tabelle 24 Frage 11: Werden die Projektergebnisse im Unternehmen wirtschaftlich verwertet? Im Bundesländervergleich

Tabelle 25 Frage 11: Werden die Projektergebnisse im Unternehmen wirtschaftlich verwertet? Nach Beschäftigtengrößenklassen

Tabelle 26 Frage 11: Werden die Projektergebnisse im Unternehmen wirtschaftlich verwertet? Nach ÖNACE Klassifikation der Projekte ... 54

Tabelle 27 Kooperationsverhalten im Bundesländervergleich ............................55

Tabelle 28 Kooperationsverhalten nach Beschäftigtengrößenklassen ................56

Tabelle 29 Kooperationsverhalten nach ÖNACE Klassifikation der Projekte ......57 


\section{Grafikverzeichnis}

Grafik 1 Erreichung der Projektziele aus technischer und wirtschaftlicher Sicht, 2009 abgeschlossene Projekte

Grafik 2 Erreichung der Projektziele aus technischer und wirtschaftlicher

Sicht, abgeschlossene Projekte der Jahre 2007-2009.

Grafik 3 Erreichung der Projektziele aus wirtschaftlicher Sicht nach

Programmtyp, abgeschlossene Projekte der Jahre 2007-2009, in Prozent.

Grafik 4 Wirtschaftliche Verwertung der Projektergebnisse der abgeschlossenen Projekte im Zeitverlauf, vergebenes Fördervolumen in Mio. $€$

Grafik 5 Direkte wirtschaftliche Ergebnisse der Befragungsteilnehmer im Zeitverlauf, in Mio $€$

Grafik 6 Fördermultiplikatoren der Jahre 2007-2009

Grafik 7 Beschäftigungseffekte der im Jahr 2009 abgeschlossenen Projekte nach Beschäftigtengrößenklassen der Unternehmen, Anzahl.

Grafik 8 Entwicklung der Beschäftigtenzahl in Unternehmen mit und ohne wirtschaftlicher Zielerreichung aus den FFG-geförderten Projekten

Grafik 9 Entwicklung der Beschäftigtenzahl in Unternehmen mit wirtschaftlicher Zielerreichung aus den FFG-geförderten Projekten, nach Unternehmensgrößenklassen

Grafik 10 Fördervolumen der abgeschlossenen Projekte mit bzw. ohne wirtschaftlicher Zielerreichung aus den FFG-geförderten Projekten, nach Größenklassen und Form der Zuwendung

Grafik 11 Arten der Innovation von abgeschlossenen Projekten, 2007-2009.... 37

Grafik 12 Hätten Sie das Projekt auch ohne Förderung durchgeführt? Jahr 2009

Grafik 13 Hätten Sie das Projekt auch ohne Förderung durchgeführt? Abgeschlossene Projekte der Jahre 2007-2009 


\section{Das Wichtigste in Kürze}

Im Wirkungsmonitoring der im Jahr 2009 abgeschlossenen Projekte konnte ein Höchststand von 498 Projektteilnahmen durch 406 Unternehmen bzw. eine Rücklaufquote von $77,6 \%$ erreicht werden. Es setzte sich der Trend der letzten Jahre insofern fort, als vermehrt Projekte über das Basisprogramm hinaus erfasst wurden. 130 Projekte konnten den „sonstigen“ FFG Programmen zugeordnet werden.

Während Basisprogrammprojekte der experimentellen Entwicklung mit dem unmittelbaren Ziel einer wirtschaftlichen Verwertung zuzurechnen sind, stellen die sonstige Programme besonders auf den Aufbau von Forschungsinfrastruktur sowie ein breites Portfolio von gesellschaftsrelevanten Zielen ab, bzw. weisen vermehrt vorwettbewerblichen Charakter auf. Deshalb zeigen Projekte der sonstigen Programme erwartungsgemäß vier Jahre nach Projektende geringere konkrete wirtschaftliche Auswirkungen in Unternehmen, jedoch höhere Auswirkungen bei den „weicheren“ Innovationsfeldern bzw. -indikatoren.

Zum Beispiel resultieren Projekte des Basisprogramms vermehrt in Produkt- und Verfahrensinnovationen. Die Vergleichsgruppe der sonstigen Programme hat ebenfalls einen Schwerpunkt bei Produktinnovationen, aber einen relativ höheren Anteil an neuen und veränderten Dienstleistungen sowie organisatorischen Innovationen.

Projekte des Basisprogramms und sonstigen Programme weisen auch etwas unterschiedliche Kooperationsmuster auf. Während jene des Basisprogramms tendenziell stärker nationale und internationale Kooperationen (EU und darüber hinaus) entlang der Wertschöpfungskette sowie mit vornehmlich Universitäten eingehen, sind jene der sonstigen Programme teilweise ebenfalls auf europäischer Ebene angesiedelt, aber tendenziell stärker auf nationale und regionale Kooperationen mit einem noch deutlicheren Schwerpunkt auf das wissenschaftliche System abgestellt.

Patentneuanmeldungen wurden in $33 \%$ der untersuchten Projekte eingereicht, wobei diese eine Summe von 323 Schutzrechten bilden. Darüber hinaus wurden die Ergebnisse von 166 Projektteilnahmen wissenschaftlich publiziert. Während der Anteil der Patentanmeldungen pro Projekt im Basisprogramm erwartungsgemäß höher ist $(0,81$ vs. 0,17$)$, resultieren die sonstigen Programme in einen höheren Anteil an wissenschaftlichen Publikationen pro Projekt (bei $28 \%$ vs. $47 \%$ der Projekte).

Einen Projekterfolg aus technischer Sicht konnten knapp 9 von 10 Projekten erzielen. Etwas weniger als zwei Drittel der geförderten Projekte (62 \%) weisen auch einen unmittelbaren wirtschaftlichen Erfolg aus. Damit knüpfen die abgeschlosse- 
nen Projekte des Jahres 2009 nahtlos an das hohe Niveau der Vorjahre an. Diese Erfolgsquoten sind unabhängig von den beiden Programmtypen (Basisprogramm und sonstige Programme).

In Summe haben $52 \%$ der Projekte (260) positive Wirkungen auf die Erhaltung bzw. Schaffung von Arbeitsplätzen entfalten können. Eine Netto-Betrachtung der Entwicklung der Beschäftigtenzahl in Unternehmen mit bzw. ohne Erreichung der wirtschaftlichen Projektziele zeigt, dass FFG geförderte KMU, die bereits einen wirtschaftlichen Erfolg melden konnten, auch ihre Beschäftigung nach Projektende relativ deutlich steigern konnten, während sich KMU ohne Projekterfolg deutlich negativ entwickelten. Interessanterweise entwickelten sich Großunternehmen ohne Projekterfolg ebenso negativ, während jene mit Projekterfolg ihren Mitarbeiter/innenstand nahezu halten konnten. Bei Großunternehmen ist jedoch aufgrund inres teilweise großen Forschungsportfolios die Zuordnung der Wirkungen auf ein FFG-Projekt naturgemäß schwieriger als bei KMU.

Beschäftigungseffekte für FuE-Mitarbeiter/innen während bzw. nach der Projektlaufzeit lassen sich bei 255 der insgesamt 498 Projekte (51\%) feststellen, wobei diese auf externe und interne Rekrutierung zurückzuführen sind. Im Zuge von 180 Projekten (36\%) kam es zu externen Rekrutierungen; zusätzlich gaben 143 Befragte $(29 \%)$ an, interne Personalneuzuteilungen getroffen zu haben.

Die Zahl der FuE-Mitarbeiter/innen, die während der Projektdurchführung extern rekrutiert wurden beläuft sich auf 454, während 335 nach Abschluss eingestellt wurden. Interne Personalrocharden betrafen 198 Mitarbeiter/innen während der Projektdurchführung bzw. 105 Mitarbeiter/innen nach Abschluss des Projekts.

Obwohl aufgrund der hohen Projektzahl im Jahr 2009 absolut höhere Arbeitsplatzeffekte gegenüber den Vorjahren gemeldet wurden, stellt dies relativ betrachtet eine Abschwächung dar. Da sich 2009 die Wirtschaftskrise entfaltete, ist ein Zusammenhang mit diesem Ergebnis zu vermuten. Insbesondere große Unternehmen haben trotz einer erfolgreichen Innovation eher verhalten mit Neuanstellungen reagiert.

Für $9 \%$ der Unternehmen stellte das geförderte Projekt die erste FuE-Tätigkeit dar. Für den Großteil dieser Unternehmen, d. h. 7 \%-Punkte, wirkte das geförderte Projekt darüber hinaus als Anstoß für weitere FuE Projekte. Dies bestätigt die Ergebnisse der letzten Jahre.

Neukunden für die FFG leiten einen relativ höheren Nutzen hinsichtlich der „weichen" Innovationsfaktoren aus der FFG Forschung ab (neue Kooperation, etc.), aber hinsichtlich der kommerziellen Verwertung der Forschungsergebnisse liegen sie hinter den Bestandskunden, die mehr Erfahrung mit derartigen Projekten aufweisen. 
$23 \%$ der Projekte wären ohne Förderung nicht durchgeführt worden, $4 \%$ unverändert und $15 \%$ in überwiegendem Ausmaß, wobei sich die Additionalität der Projektdurchführung bei den sonstigen Programmen besonders hoch darstellt. Generell gilt auch: je kleiner das Unternehmen, desto geringer die Bereitschaft der Projektdurchführung ohne Fördermittel; ähnliches kann für FFG-Neukunden formuliert werden. 


\section{Einleitung}

Das Wirkungsmonitoring des Jahres 2013 bezieht sich auf alle von der FFG geförderten Projekte, in denen Unternehmen als Projektträger oder Partner auftreten und die im Jahr 2009 abgeschlossen wurden. Indem Projekte vier Jahre nach dem formalen Projektende in die Analyse einbezogen werden, sollen Aussagen über die mittelfristigen Auswirkungen der eingesetzten Fördermittel ermöglicht werden. Darüber hinaus wird in diesem Jahr auch eine Längsschnittanalyse für die Jahre 2007-2009 durchgeführt.

Ab dem Jahr 2010 (Projektende 2006) wurde das Wirkungsmonitoring über das Basisprogramm (BP) hinaus auf andere Programme der FFG ausgeweitet, sofern nicht bereits aufgrund der relativen Bedeutung von bestimmten Programmen ein eigenes Wirkungsmonitoring bestand. Unternehmensprojekte im Rahmen von z.B. K-Zentren, AplusB, aber auch personenbezogene Programme sind mit diesem Wirkungsmonitoring nicht abgedeckt. In den letzten beiden Jahren konnte erstmals eine substantiellere Anzahl an "sonstigen“ Programmen (thematische und Strukturprogramme, ALR) im Sample erfasst werden, das spezifischere Aussagen zu den sonstigen Programmen erlaubt.

Die in den "sonstigen“ Programmen zusammengefassten Projekte weisen je nach Programm heterogene und meist auch andere Wirkungslogiken als Basisprogrammprojekte auf. Während Basisprogrammprojekte der experimentellen Entwicklung mit dem unmittelbaren Ziel einer wirtschaftlichen Verwertung zuzurechnen sind, stellen die sonstige Programme besonders auf den Aufbau von Forschungsinfrastruktur sowie einem breiten Portfolio von gesellschaftsrelevanten Zielen ab, bzw. weisen vermehrt vorwettbewerblichen Charakter auf.

An dieser Stelle soll darauf hingewiesen werden, dass anhand des Analysedesigns keine allumfassende quantitative Erfassung der Nettoeffekte möglich ist, da sich schwer fassbare Nebeneffekte (Substitution, Leverage, etc.) positiv wie auch negativ auf die hier ermittelten Resultate auswirken können. Die Erfassung dieser Effekte wäre mit einem unverhältnismäßig hohen Aufwand (und Budget) verbunden. Das jährlich umgesetzte Wirkungsmonitoring legt den Schwerpunkt vielmehr auf die Analyse von deskriptiven Daten und qualitativen Inhalten und analysiert diese im Detail, um ein umfassenderes Bild potenzieller Wirkungen auf die teilnehmenden Unternehmen zu erhalten.

Eine analytisch-quantitative Schätzung von Effekten wurde im Jahr 2004 durch eine einmalige Studie eines Konsortiums vorgenommen, die im Wesentlichen die positiven Ergebnisse des Wirkungsmonitorings über die Jahre bestätigen konnte (Schibany et al. 2004; Evaluation FFF - Impact Analysis. Background report 3.2. Joanneum Research, INTEREG research report series. Vienna, Austria). 


\section{Methodik und Eckdaten der analysierten Unternehmen}

Das Wirkungsmonitoring basiert auf einer standardisierten Unternehmensbefragung sowie der FFG Projektdatenbank. Der in der Erhebung verwendete Fragebogen wird den Fördernehmern (Unternehmen) durch die FFG zugesandt. Die beantworteten Fragebögen werden an die KMU Forschung Austria retourniert und entsprechend ausgewertet.

Bei der Betrachtung der eingegangenen Fragebögen ist zu berücksichtigen, dass die Zahl der verwertbaren Fragebögen (498) von der Zahl der geförderten Unternehmen (406) abweicht. Eine Unterscheidung ist diesbezüglich erforderlich, da ein Unternehmen mehrere Projekte zeitgleich durchführen kann. Des Weiteren können Projekte auch in Form von Kooperationen abgewickelt werden, wodurch Wirkungen der Forschungsaktivitäten nicht auf einen einzelnen Fördernehmer beschränkt sein müssen und Angaben zu ein und demselben Projekt für das jeweils eigene Unternehmen erfolgen können.

Im Wirkungsmonitoring wird in der Folge mit „Projekt" das Projekt / der Projektteil in Bezug auf ein Unternehmen bezeichnet, d. h. bei Kooperationsprojekten wird der Projektteil je Unternehmen herangezogen, nicht das gesamte Kooperationsprojekt aggregiert. Dies entspricht damit der Anzahl der ausgewerteten Fragebögen.

Der Querschnittscharakter der Befragung beinhaltet insofern Verzerrungen, als größere Innovationen oft in „Projektfamilien“ vorangetrieben werden, anhand derer konsekutive Projekte mit unterschiedlichen Schwerpunkten und Finanzierungsstrukturen umgesetzt werden. Davon werden mehr oder weniger große Teile von der FFG gefördert. Ein KMU kann z.B. aufgrund eines singulären von der FFG geförderten Projekts eine Innovation am Markt lancieren. Am anderen Ende des Spektrums hat ein technologieorientiertes globales Unternehmen laufend mehrere FuE Projekte parallel laufen. Dabei wird eine Zuordnung von wirtschaftlichen Effekten zu einem FFG Projekt oft schwer. Dies ist auch der Grund dafür, dass Großunternehmen öfters keine wirtschaftlichen Effekte im Rahmen des Wirkungsmonitorings angeben. Trotzdem kann es vorkommen, dass das geförderte Projekt eines Großunternehmens eine Core-Technology hinsichtlich Produktentwicklung betrifft, mit der fallweise ein hoher Umsatz am Markt erwirtschaftet werden kann. Aus diesem Grund wird bei Unternehmen, die sehr hohe wirtschaftliche Effekte im Rahmen des Wirkungsmonitorings angeben, der Zusammenhang mit der FFG Förderung mit der KMU Forschung Austria per Telefon noch erörtert. 
Dennoch werden zwecks einer kontinuierlichen Darstellung der FFG Wirkungen in der Breite die erfolgreichsten Projekte im Sinne von statistischen Ausreißern hinsichtlich ihrer wirtschaftlichen Wirkungen nicht in die Analysen einbezogen, da diese größere Schwankungen der Ergebnisse über die Jahre implizieren würde. Anders formuliert bedeutet dies, dass es über die in diesem Bericht dargestellten Wirkungen hinaus noch singuläre Fälle gibt, die große Wirkungen erzielten, aber aufgrund einer konsistenten Darstellung über die Jahre nicht aufgenommen wurden.

Im Jahr 2009 konnten insgesamt 1.021 Projekte von Unternehmen formal abgeschlossen werden (Basisprogramm und sonstige Programme). Im Zentrum der nachfolgenden Betrachtung stehen jedoch nur all jene Projekte, die ein Projektvolumen von mindestens $€ 20.000$,- aufweisen und keine reinen Feasibility Studien darstellen. Schließlich wurden 642 Fragebögen an derzeit bestehende Unternehmen versendet (mit zweimaliger Erinnerung), welche in 502 Rückmeldungen mündeten. Das entspricht einer sehr hohen Brutto-Rücklaufquote von rd. 78,5\%. ${ }^{1}$ Die Netto-Rücklaufquote, welche den Ausgangswert für die nachfolgende Analyse bildet, liegt bei $77,6 \%$ und setzt sich aus 498 verwertbaren Fragebögen zusammen.

Die Fördernehmer des Basisprogramms (inkl. Headquarterprogramm) stellen die traditionell größte Gruppe der Antwortenden dar, und weisen mit einer Rücklaufquote von $79,1 \%$ eine überdurchschnittlich hohe Auskunftsfreudigkeit auf. Die restlichen, sonstigen Programme kommen durchschnittlich auf eine Rücklauf von $73,4 \%$ und sind zu über zwei Drittel auf die Programme IV2S, BRIDGE und protec-net zurückzuführen.

Projekte des Programms BRIDGE gehören zwar zum Bereich Basisprogramme, werden aber aufgrund ihres Fokus auf den Brückenschlag zwischen Grundlagenund Anwendungsforschung, und die damit einhergehende größere Entfernung von konkreten Produktentwicklungen und einer etwaigen Marktreife, bei spezifischen Auswertungen im Wirkungsmonitoring den sonstigen Programmen zugeordnet.

\footnotetext{
${ }^{1}$ Bezogen auf alle geförderten, noch bestehenden Unternehmen. Eine zweite Erinnerung wurde heuer versendet, da sich die erste Aussendung mit der CIS-Erhebung der Statistik Austria überkreuzte.
} 
Tabelle 1 Verteilung der in die Analyse einbezogenen, sowie 2009 abgeschlossenen Projekte nach Programmen

\begin{tabular}{|c|c|c|c|c|c|}
\hline Bereich & Programm & Versendet & Ausgewertet & $\begin{array}{l}\text { Netto- } \\
\text { Rücklauf }\end{array}$ & $\begin{array}{l}\text { Anteil an aus- } \\
\text { gewerteten } \\
\text { Fragebögen }\end{array}$ \\
\hline \multirow{2}{*}{ BP } & Basisprogramm & 465 & 368 & $79,1 \%$ & $73,9 \%$ \\
\hline & BRIDGE & 43 & 32 & $74,4 \%$ & $6,4 \%$ \\
\hline \multirow{8}{*}{ TP } & IV2S & 57 & 39 & $68,4 \%$ & $7,8 \%$ \\
\hline & TAKE OFF & 11 & 9 & $81,9 \%$ & $1,8 \%$ \\
\hline & NAWI & 6 & 4 & $66,7 \%$ & $0,8 \%$ \\
\hline & $\begin{array}{l}\text { Energie der } \\
\text { Zukunft }\end{array}$ & 2 & 2 & $100 \%$ & $0,4 \%$ \\
\hline & IV2Splus & 2 & 2 & $100 \%$ & $0,4 \%$ \\
\hline & KIRAS & 2 & 2 & $100 \%$ & $0,4 \%$ \\
\hline & NANO & 2 & 2 & $100 \%$ & $0,4 \%$ \\
\hline & $\begin{array}{l}\text { Neue Energien } \\
2020\end{array}$ & 2 & 2 & $100 \%$ & $0,4 \%$ \\
\hline \multirow{3}{*}{ SP } & protec-net & 30 & 19 & $63,3 \%$ & $3,8 \%$ \\
\hline & CIR-CE & 2 & 2 & $100 \%$ & $0,4 \%$ \\
\hline & REGplus & 1 & 1 & $100 \%$ & $0,2 \%$ \\
\hline \multirow{3}{*}{ ALR } & ASAP & 12 & 10 & $83,3 \%$ & $2,0 \%$ \\
\hline & Artist & 5 & 4 & $80 \%$ & $0,8 \%$ \\
\hline & Gesamt & 642 & 498 & $77,6 \%$ & $100,0 \%$ \\
\hline
\end{tabular}

Quelle: KMU Forschung Austria; BP = Basisprogramme, TP = Thematische Programme, SP = Strukturprogramme, ALR = Agentur für Luft- und Raumfahrt

Der Anteil der Programme außerhalb der BP ist kontinuierlich gestiegen. Wurde 2011 noch vorwiegend das Basisprogramm hinsichtlich seiner Wirkungseffekte untersucht (89\% der FuE-Projekte), so sind es heuer noch knapp drei Viertel der befragten Fördernehmer. Dies ist jedoch nur eine relative Verschiebung. Wie der untenstehenden Tabelle zu entnehmen ist, konnte absolut ein deutlicher Anstieg der auszuwertenden Fragebögen erzielt werden. Der Grund dafür liegt im höheren Rücklauf, und insbesondere der höheren Zahl an Förderfällen im Jahr 2009. 
Tabelle 2 Verteilung der in die Analyse einbezogenen, sowie in den Jahren 20072009 abgeschlossenen Projekte, Anzahl

\begin{tabular}{|l|r|r|r|}
\hline & $\mathbf{2 0 0 7}$ & $\mathbf{2 0 0 8}$ & $\mathbf{2 0 0 9}$ \\
\hline Basisprogramm & 262 & 243 & 368 \\
\hline sonstige Programme & 34 & 69 & 130 \\
\hline \hline Gesamt & 296 & 312 & 498 \\
\hline
\end{tabular}

Quelle: KMU Forschung Austria

Die höhere Zahl unterschiedlicher Programmtypen erlaubt zunehmend spezifischere Aussagen hinsichtlich der Wirkungseffekte der sonstigen Programme. Grundsätzlich werden im Wirkungsmonitoring die Auswertungen über alle untersuchten Projekte aggregiert dargestellt und nur bei signifikanten Unterschieden nach Basisprogramm und sonstigen Programmen unterschieden.

Die Klassifikation der durchgeführten Projekte hinsichtlich des angewandten Förderinstruments wird in diesem Berichtsjahr 2013 erstmals vorgenommen. Diese Darstellung kann als eine weitere Ausdifferenzierung der sonstigen Programme gewertet werden, da die 368 Projekte des Basisprogramms zur Gänze im Bereich der Einzelprojekte Experimentelle Entwicklung zugerechnet sind. Von den restlichen, „sonstigen“ Programmen wurden kooperative FuE-Projekte, Wissenschaftstransfers und Innovationsnetzwerke am häufigsten in Anspruch genommen. Auf Grund der nahezu identen Unternehmensverteilung zwischen den angewandten Instrumenten und der Einteilung in BP bzw. sonstige Programme, wird auf weiterführende Vergleiche der Instrumentengruppen verzichtet.

Tabelle 3 Zuordnung der Projekte zur neuen FFG Klassifikation der Förderinstrumente

\begin{tabular}{|l|c|c|}
\hline Förderinstrument & Anzahl & $\%$ \\
\hline Einzelprojekt EE & 371 & $75 \%$ \\
\hline Kooperative FuE- Projekte & 58 & $12 \%$ \\
\hline Wissenschaftstransfer & 32 & $6 \%$ \\
\hline Innovationsnetzwerke & 21 & $4 \%$ \\
\hline Sondierung & 6 & $1 \%$ \\
\hline Einzelprojekt IF & 4 & $1 \%$ \\
\hline Qualifizierungsmaßnahme & 3 & $1 \%$ \\
\hline Sonstige & 3 & $1 \%$ \\
\hline \hline Gesamt & 498 & $100 \%$ \\
\hline
\end{tabular}

Quelle: FFG und KMU Forschung Austria; EE = Experimentelle Entwicklung; IF = Industrielle Forschung 
Wie bereits in den Vorjahren wird eine Unterscheidung zwischen Projekten mit marktnahem (experimentelle Entwicklung) und marktfernem (industrielle Forschung) Charakter vorgenommen, die von der FFG vorgenommen wurde. Im Zuge der diesjährigen Auswertung kann zwischen 75 marktfernen (15\%) und 423 marktnahen Projekten (85\%) unterschieden werden. Die marktfernen Projekte sind hierbei zur Gänze den sonstigen Programmen zuzuordnen, welche einen Anteil von rd. 58 \% ausmachen. Die übrigen 55 Projekte der sonstigen Programme (42 \%) weisen daher eher marktnahe Eigenschaften auf (2007: 59 \%; 2008: 50 \%).

Die Klassifikation der befragten Unternehmen hinsichtlich ihrer Größenklasse nach EU-Definition ${ }^{2}$ wurde im Vorjahr erstmalig durchgeführt. Die von der FFG zur Verfügung gestellte Eingliederung der geförderten Unternehmen hinsichtlich ihrer Größenklasse erfolgt nach den Kriterien Beschäftigtenzahl, Umsatz, Bilanzsumme und Eigenständigkeit. Rund $41 \%$ (= 206) der geförderten Projekte wurden von Großunternehmern (GU) durchgeführt, gefolgt von den Kleinst- und Kleinunternehmen (KU: 29 \% bzw. 143 Projekte) und mittlere Unternehmen (MU: 26\% bzw. 130 Projekte). In den 18 verbliebenen Fällen war eine Zuordnung zu einer der genannten Kategorien nicht möglich. Abweichungen dieser Unternehmensverteilung und der Verteilung nach dem Kriterium der Mitarbeiterzahl sind nicht nur auf Umsatz und Bilanzsumme zurückzuführen, sondern in umfangreichen Ausmaß auch der Eigentümerstruktur der Unternehmen geschuldet. Damit gibt es einen recht bedeutenden Anteil an GU, die nach ihrer Mitarbeiter- sowie FuE-Mitarbeiterzahl eher mittelständischen Charakter aufweisen.

Insgesamt sind mehr als 161.000 Mitarbeiter/innen in den 406 befragten Unternehmen beschäftigt. Davon sind 16.150 FuE-Mitarbeiter/innen, wovon 4.518 an den 498 untersuchten, FFG-geförderten Projekten arbeiteten; 494 bzw. $11 \%$ der FuE-Mitarbeiter sind weiblich. Im Median waren fünf FuE-Mitarbeiter/innen an einem Projekt beteiligt (Mittelwert: 9,5). Mittelwert und Median liegen beim Basisprogramm (11 bzw. 5 FuE-Mitarbeiter/innen) über den Werten der sonstigen Programme (6 bzw. 4 FuE-Mitarbeiter/innen). Im Vergleich zu den Vorjahren ist die Zahl der FuE-Mitarbeiterinnen zwar absolut gestiegen (2007: 357, 2008: 401), jedoch ist der durchschnittliche Anteil rückläufig. Die FuE-Personalintensität (Anteil der FuE-Mitarbeiter/innen an Gesamtbeschäftigten) ist bei $\mathrm{KU}$ signifikant am höchsten (Median: $33 \%$ ), gegenüber $16 \%$ bei MU und $9 \%$ bei GU.

Von den im Jahr 2009 abgeschlossenen Projekten wurden 20 (4 \%) unter weiblicher Projektleitung durchgeführt, wobei sechs Projekte den sonstigen Programmen zuzuordnen sind. Gegenüber den Vorjahren ist dies sowohl in absoluten als auch relativen Werten ein Rückgang (2007: 21 bzw. 7 \% 2008: 29 bzw. 9 \%).

${ }^{2}$ Definition der Größenklassen siehe Anhang 
Hauptsächlich sind Projektleiterinnen in Kleinst- und Kleinunternehmen eingesetzt worden (45\%, gesamt rd. $29 \%$ ) bzw. haben sie Projekte alleine durchgeführt (65\%, gesamt rd. $60 \%$ ).

Auf Grund der geringen Anzahl an Projektleiterinnen sind weiterführende Aussagen bzw. Ergebnisbeschreibungen für Projekte unter weiblicher Leitung nicht möglich.

Die Verteilung der 2009 abgeschlossenen Projekte nach Beschäftigtengrößenklassen der Unternehmen ist in der untenstehenden Tabelle zusammengefasst. Wie bereits in den beiden Vorjahren stellen die Unternehmen mit 1 bis 20 Mitarbeiter/innen (26\%) den größten Anteil der Befragten dar (2007: 34 \%, 2008: $21 \%$ ). Verhältnismäßig hoch ist hierbei der Anteil von Projekten, die den sonstigen Programmen zuzuordnen sind (38 \%). In den Größenklassen 101 bis 250 Beschäftigte $(17 \%)$ sowie über 1.000 Mitarbeitern (15\%) ist in etwa ein weiteres Drittel der Unternehmen konzentriert. Wesentlichen Anteil daran haben zum einen die BRIDGE-Projekte in der Größenklasse von 1 bis 20 Beschäftigten (11 Projekte) bzw. IV2S in der Größenklasse über 1.000 Beschäftigte (12 Projekte).

Tabelle 4 Verteilung der 2009 abgeschlossenen Projekte nach Beschäftigtengrößenklassen der Unternehmen

\begin{tabular}{|l|c|c|c|c|}
\hline Beschäftigtengrößenklassen & Anzahl & $\%$ & Anzahl BP & $\begin{array}{c}\text { Anzahl } \\
\text { sonstige P. }\end{array}$ \\
\hline 1 - 20 Beschäftigte & 123 & $26 \%$ & 77 & 46 \\
\hline $21-50$ Beschäftigte & 58 & $12 \%$ & 42 & 16 \\
\hline 51 -100 Beschäftigte & 50 & $11 \%$ & 43 & 7 \\
\hline 101 - 250 Beschäftigte & 77 & $17 \%$ & 60 & 17 \\
\hline 251 - 500 Beschäftigte & 46 & $10 \%$ & 40 & 6 \\
\hline $501-1.000$ Beschäftigte & 45 & $10 \%$ & 38 & 7 \\
\hline über 1.000 Beschäftigte & 69 & $15 \%$ & 46 & 23 \\
\hline \hline Gesamt & 468 & $100 \%$ & 346 & 122 \\
\hline
\end{tabular}

Quelle: KMU Forschung Austria; BP = Basisprogramm

Die Zuteilung der durchgeführten Projekte hinsichtlich thematischer Klassifikation der geförderten Unternehmen zeigt auf, dass eine Mehrzahl von Forschungsvorhaben im Bereich der Herstellung von Waren abgewickelt wurde. Wie auch in den Vorjahren liegt der Wert im Bereich von $60 \%$, wobei der Maschinenbau und die Herstellung von EDV und optischen Geräten die am häufigsten geförderten Branchen darstellen. 
Ebenfalls häufig vertreten ist die Branche der wissenschaftlichen und technischen Dienstleistungen, die immerhin noch $12 \%$ der eingegangen Fragebögen umfassen. Die Verteilung ist über die letzten drei Jahre konstant, allein die Bedeutung der wissenschaftlichen und technischen Dienstleistungen ist von $7 \%$ (2007) auf $12 \%$ gestiegen.

Tabelle 5 Verteilung der analysierten und 2009 abgeschlossenen Projekte nach ÖNACE-Klassifikation der Projekte

\begin{tabular}{|c|c|c|}
\hline Abschnitt & Anzahl & $\%$ \\
\hline A: Land- und Forstwirtschaft; B: Bergbau & 5 & $1 \%$ \\
\hline C: Herstellung von Waren & $(284)$ & $(57 \%)$ \\
\hline EDV u. optische Geräte & 64 & $13 \%$ \\
\hline Maschinenbau & 64 & $13 \%$ \\
\hline Metallerzeugnisse & 26 & $5 \%$ \\
\hline Chemische Erzeugnisse, Gummi- und Kunststoffwaren & 30 & $6 \%$ \\
\hline Elektrische Ausrüstungen & 13 & $3 \%$ \\
\hline Sonstige Waren & 87 & $17 \%$ \\
\hline $\begin{array}{l}\text { D: Abfallentsorgung/Beseitigung } \\
\text { E: Umweltverschmutzung; F: Bau }\end{array}$ & 7 & $1 \%$ \\
\hline G: Handel; Instandhaltung und Reparatur von KFZ & 34 & $7 \%$ \\
\hline J: Information und Kommunikation & 49 & $10 \%$ \\
\hline $\begin{array}{l}\text { K: Finanzdienstleistungen, L: Wohnungswesen, } \\
\text { N: Wirtschaftliche Dienstleistungen }\end{array}$ & 11 & $2 \%$ \\
\hline M: Wissenschaftliche und technische Dienstleistungen & 59 & $12 \%$ \\
\hline Sonstige Projekte & 49 & $10 \%$ \\
\hline Gesamt & 498 & $100 \%$ \\
\hline
\end{tabular}

Quelle: KMU Forschung Austria

$60 \%$ der untersuchten Projekte wurden von den jeweiligen Unternehmen alleine durchgeführt. $^{3}$ Von den $40 \%$ der in einem Konsortium durchgeführten Projekte hatten $52 \%$ die Konsortialleitung inne. Im Vergleich zum Vorjahr ist das Verhältnis zwischen alleinigen Projektdurchführungen und Konsortialprojekten nahezu konstant geblieben (2008: 59 \% bzw. 41 \%). Ein deutlicher Sprung lässt sich allerdings gegenüber dem Jahr 2007 feststellen, wo lediglich $32 \%$ der untersuchten Projekte in Kooperationen durchgeführt wurden. Dies beruht auf den zunehmenden Anteil von Projekten der thematischen Programme sowie BRIDGE in das Wirkungsmonitoring.

${ }^{3}$ Dies ist die Einschätzung der Unternehmen, die nicht immer mit der Erfassung der FFG Datenbank zusammenpasst, da die Unternehmen z. B. einen Subauftrag an eine Forschungseinrichtung als Kooperation / Konsortium sehen können. 
Tabelle 6 Verteilung der Projekte nach der Rolle des Unternehmens innerhalb des Gesamt-Projektes

\begin{tabular}{|c|c|c|}
\hline & Anzahl & $\%$ \\
\hline Vom Unternehmen allein durchgeführt & 297 & $60 \%$ \\
\hline In einem Konsortium durchgeführt & 196 & $40 \%$ \\
\hline Gesamt & 493 & $100 \%$ \\
\hline \multicolumn{3}{|l|}{ Wenn in einem Konsortium durchgeführt... } \\
\hline ... als Konsortialleiter & 96 & $52 \%$ \\
\hline ... als Konsortialpartner & 90 & $48 \%$ \\
\hline Gesamt & 186 & $100 \%$ \\
\hline
\end{tabular}

Quelle: KMU Forschung Austria

Die Durchführung von Forschungsprojekten im Bereich der sonstigen Programme unterstreicht mit knapp $85 \%$ an Konsortialprojekten die teilweise auf Kooperation ausgelegten Programmdesigns. Insgesamt wurden mehr als die Hälfte der Konsortialprojekte (56\%) außerhalb des Basisprogramms durchgeführt. Nach der Abgrenzung marktnahe/marktfern wurden rd. 81 \% (61 von 85) der marktfernen Projekte im Konsortium durchgeführt. Projekte aus den Bereichen Verkehr und Lagerei (100\%), Finanz- und Wirtschaftsdienstleistungen (64\%) sowie Wissenschaftliche und technische Dienstleistungen (63\%) weisen die höchsten Anteile an Konsortialprojekten auf.

Projekte in Form von Konsortien weisen eine technische Zielerreichung von $88 \%$ Prozent auf und liegen damit nahezu gleichauf mit den alleine absolvierten FuEProjekten $(89 \%)$. Deutlichere Unterschiede sind bezüglich des wirtschaftlichen Erfolges der Umsetzung erkennbar. Bei rd. 70 \% der allein durchgeführten Projekte wurde das Projektziel aus wirtschaftlicher Sicht erreicht; das liegt um zehn Prozentpunkte über den Konsortialprojekten (rd. 60 \%). 


\section{Positionierung der Projekte im Portfolio der Unternehmen}

Mit der Frage nach der Positionierung der untersuchten Projekte im Portfolio der jeweiligen Unternehmen will man auf Basis der strategischen Einbettung der Projekte in die Unternehmen etwaige Rückschlüsse auf die Stoßrichtung der Projekte sowie deren ursprünglich gesehen Relevanz erhalten.

Für die befragten Unternehmen stellte die Durchführung des geförderten Projektes hauptsächlich einen Baustein in bereits etablierten Aktivitätsfeldern dar. Insgesamt $63 \%$ der Entwicklungsvorhaben wurden in bestehenden Bereichen des Unternehmensportfolios ausgeführt. Gemessen an den Vorjahren liegt dieser Prozentsatz nur leicht unterhalb der Vergleichswerte (2007: 66 \%, 2008: $65 \%$ ). Ein weiteres Drittel gab an, dass die Forschungsprojekte der Anstoß für ein neues Aktivitätsfeld innerhalb des Unternehmens waren. Weder in ein bestehendes noch in neue Aktivitätsfelder fielen 15 Projekte, deren Rolle z.B. die Unterstützung in Zertifizierungsangelegenheiten war.

Tabelle 7 Verteilung der 2009 abgeschlossenen Projekte nach der Rolle des Projekts im Portfolio des Unternehmens

\begin{tabular}{|l|c|c|}
\hline & Anzahl & $\%$ \\
\hline Baustein in bestehendem Aktivitätsfeld & 310 & $63 \%$ \\
\hline Anstoß für neues Aktivitätsfeld & 168 & $34 \%$ \\
\hline Sonstiges & 15 & $3 \%$ \\
\hline \hline Gesamt & 493 & $100 \%$ \\
\hline
\end{tabular}

Quelle: KMU Forschung Austria

In den BP sind $61 \%$ der Projekte Teil bestehender Aktivitätsfelder, während es bei den sonstigen Programmen $67 \%$ sind. Ebenso: marktnahe (62\%) und marktferne Projekte $(68 \%)$. Deutlichere Unterschiede sind zwischen der Unternehmensgrößenklassen feststellbar. Während Kleinunternehmen die geförderten Projekte häufiger als Anstoß für ein neues Aktivitätsfeld nutzen (40\%), sind $70 \%$ der Innovationstätigkeiten von Großunternehmen in bestehenden Aktivitätsfeldern angesiedelt (Mittlere Unternehmen 59 \%).

Bezogen auf die Bedeutung des Projektes innerhalb der FuE-Aktivitäten der jeweiligen Unternehmen ist festzustellen, dass für $9 \%$ das geförderte Projekt die erste FuE-Tätigkeit darstellte. In $2 \%$ der Fälle war dies auch die bisher einzige Innovationsentwicklung, d.h. die restlichen $7 \%$ der Unternehmen haben das geförderte Projekt als Anstoß für weitere FuE Projekte genommen (2008: $7 \%$; 2007: $3 \%$ ). 
Die Masse der Befragten nutzte die FFG-Förderung für den Ausbau von Forschungsprojekten im Rahmen der bestehenden Aktivitäten. Dieser Wert entspricht dem Wert des Vorjahres (88\%) bzw. liegt er leicht über dem Anteil der abgeschlossenen Projekte des Jahres 2007 (85\%).

Tabelle 8 Verteilung der 2009 abgeschlossenen Projekte nach der Rolle des Projekts hinsichtlich der FuE Aktivitäten im Unternehmen

\begin{tabular}{|l|c|c|}
\hline & Anzahl & $\%$ \\
\hline $\begin{array}{l}\text { Erste FuE Aktivität im Unternehmen (bis zum Zeit- } \\
\text { punkt der Projektdurchührung) }\end{array}$ & 47 & $9 \%$ \\
\hline Einzige FuE Aktivität im Unternehmen (bis heute) & 10 & $2 \%$ \\
\hline Ausbau von bestehenden Aktivitäten & 438 & $88 \%$ \\
\hline \hline Gesamt & 495 & $100 \%$ \\
\hline
\end{tabular}

Quelle: KMU Forschung Austria

Einen Projekterfolg aus technischer Sicht konnten knapp 9 von 10 Projekten erzielen. Dass die technisch Zielerreichung den wirtschaftlichen Erfolg nicht gewährleistet, lässt sich aus Grafik 1 ableiten. Etwas weniger als zwei Drittel der geförderten Projekte weisen auch einen unmittelbaren wirtschaftlichen Erfolg aus. 136 der Unternehmen; die aus wirtschaftlicher Sicht das Projektziel nicht erreichten, konnten dies begründen. Hauptsächlich wurden zu hohe Kosten des Endproduktes (31 Nennungen, $23 \%$ ) und die Markt- bzw. Nachfragesituation (30 Nennungen, 22 \%) genannt. Darüber hinaus gaben 22 der Befragten (16\%) an, das technische Projektziel noch nicht erreicht bzw. die geplante Verwertung noch nicht durchgeführt zu haben (5 Nennungen, $4 \%$ ). Zusätzliche Investitionen werden im Zuge von 7 Projekten benötigt (5\%), bzw. gaben 41 Befragte andere, diverse, Gründe für das Verfehlen des wirtschaftlichen Projektziels an (30\%). 
Grafik 1 Erreichung der Projektziele aus technischer und wirtschaftlicher Sicht, 2009 abgeschlossene Projekte

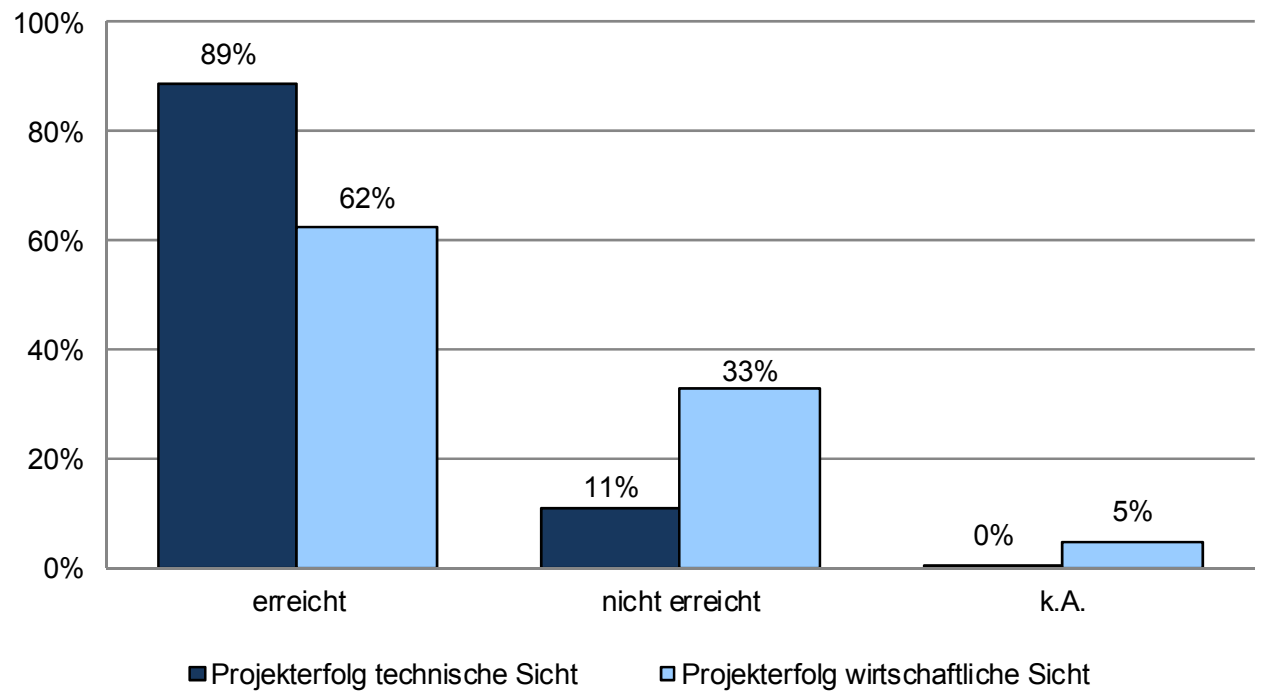

Quelle: KMU Forschung Austria, N = 498.

Wie bereits in den vergangenen Jahren liegt der Wert der technischen Projektzielerreichung auf einem konstant hohen Niveau. Aus der untenstehenden Grafik lässt sich die gleichbleibend hohe Erfüllung der Projektziele ablesen. Die häufigsten Gründe für das Nichterreichen technischer und wirtschaftlicher Projektziele waren fehlende Ressourcen, technische Umsetzbarkeit bzw. mangelnde Marktakzeptanz/ Nachfrage und ausstehende Weiterentwicklungen.

Leichte Unterschiede im Zeitverlauf gibt es hingegen bei der wirtschaftlichen Projektzielerreichung, wobei der Schwankungskorridor lediglich fünf Prozentpunkte beträgt. 
Grafik 2 Erreichung der Projektziele aus technischer und wirtschaftlicher Sicht, abgeschlossene Projekte der Jahre 2007-2009

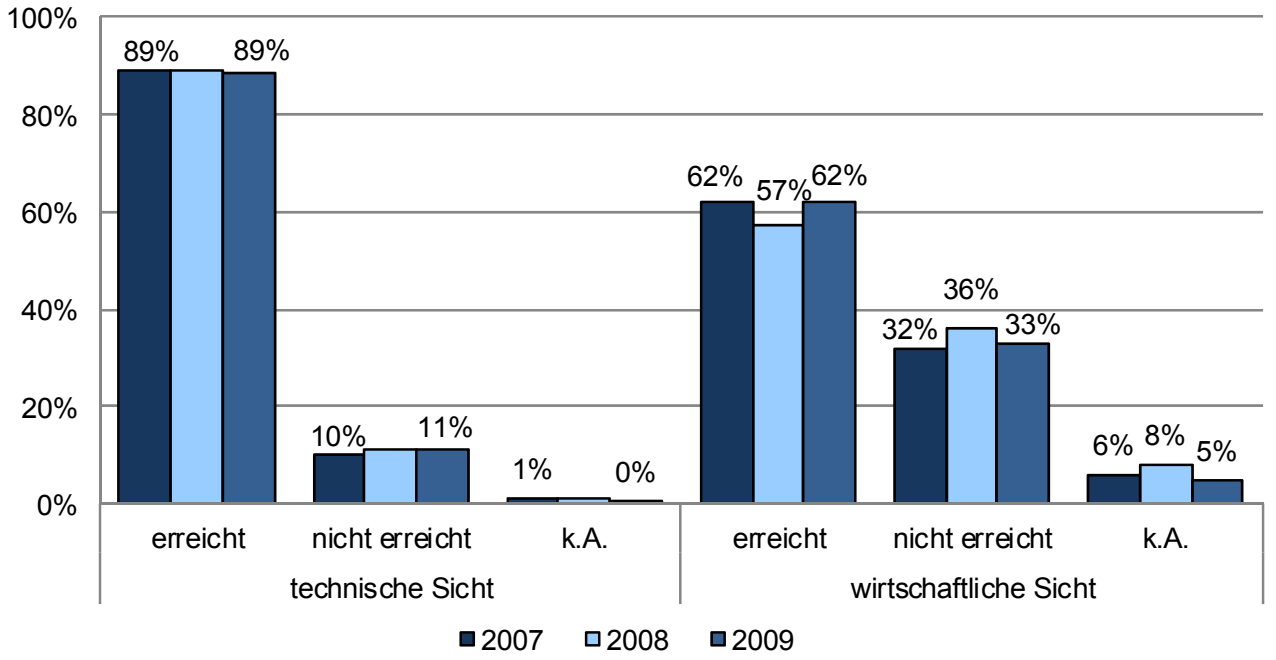

Quelle: KMU Forschung Austria, N = 296, 312, 498 .

Wie die folgende Tabelle zeigt, ist der technische und wirtschaftliche Erfolg der geförderten Projekte im Durchschnitt unabhängig von den beiden definierten Programmtypen, obwohl innerhalb der sonstigen Programme durchaus eine gewisse Heterogenität herrscht (siehe unten).

Tabelle $9 \quad$ Erreichung des Projektzieles aus technischer bzw. wirtschaftlicher Sicht nach Basisprogramm und sonstigen Programmen, 2009 abgeschlossene Projekte

\begin{tabular}{|c|c|c|c|c|c|}
\hline & \multicolumn{4}{|c|}{ Programmtyp } \\
\hline & & \multicolumn{2}{|c|}{ Basisprogramm } & \multicolumn{2}{|c|}{ sonstige Programme } \\
\hline & & Anzahl & $\%$ & Anzahl & $\%$ \\
\hline \multirow{3}{*}{$\begin{array}{l}\text { Projekterfolg aus techni- } \\
\text { scher Sicht }\end{array}$} & ja & 324 & $88 \%$ & 117 & $88 \%$ \\
\hline & nein & 42 & $11 \%$ & 13 & $12 \%$ \\
\hline & k.A. & 2 & $1 \%$ & 0 & \\
\hline \multirow{3}{*}{$\begin{array}{l}\text { Projekterfolg aus wirt- } \\
\text { schaftlicher Sicht }\end{array}$} & ja & 227 & $62 \%$ & 83 & $64 \%$ \\
\hline & nein & 122 & $33 \%$ & 42 & $32 \%$ \\
\hline & k.A. & 19 & $5 \%$ & 5 & $4 \%$ \\
\hline
\end{tabular}

Quelle: KMU Forschung Austria, N = 498; k.A. = keine Angabe

Auch im Zeitverlauf lassen sich bei der technischen Umsetzbarkeit keine Unterschiede zwischen den Programmtypen feststellen (keine Darstellung). 
Die Entwicklung des wirtschaftlichen Projekterfolges ist hingegen etwas volatiler. Bei den BP-Projekten pendelt der Wert nach wie vor um die $60 \%-M a r k e$, die sonstigen Programme können hingegen einen sprunghaften Anstieg von $50 \%$ auf über $60 \%$ verzeichnen. Dies ist auf die heuer höheren Erfolgsquoten hinsichtlich der wirtschaftlichen Ziele der Projekte aus IV2S und BRIDGE zurückzuführen, welche in IV2S vor allem von Großunternehmen, aber durchaus auch kleineren Unternehmen, und in BRIDGE mehrheitlich von kleineren Unternehmen getragen wurden.

Grafik 3 Erreichung der Projektziele aus wirtschaftlicher Sicht nach Programmtyp, abgeschlossene Projekte der Jahre 2007-2009, in Prozent

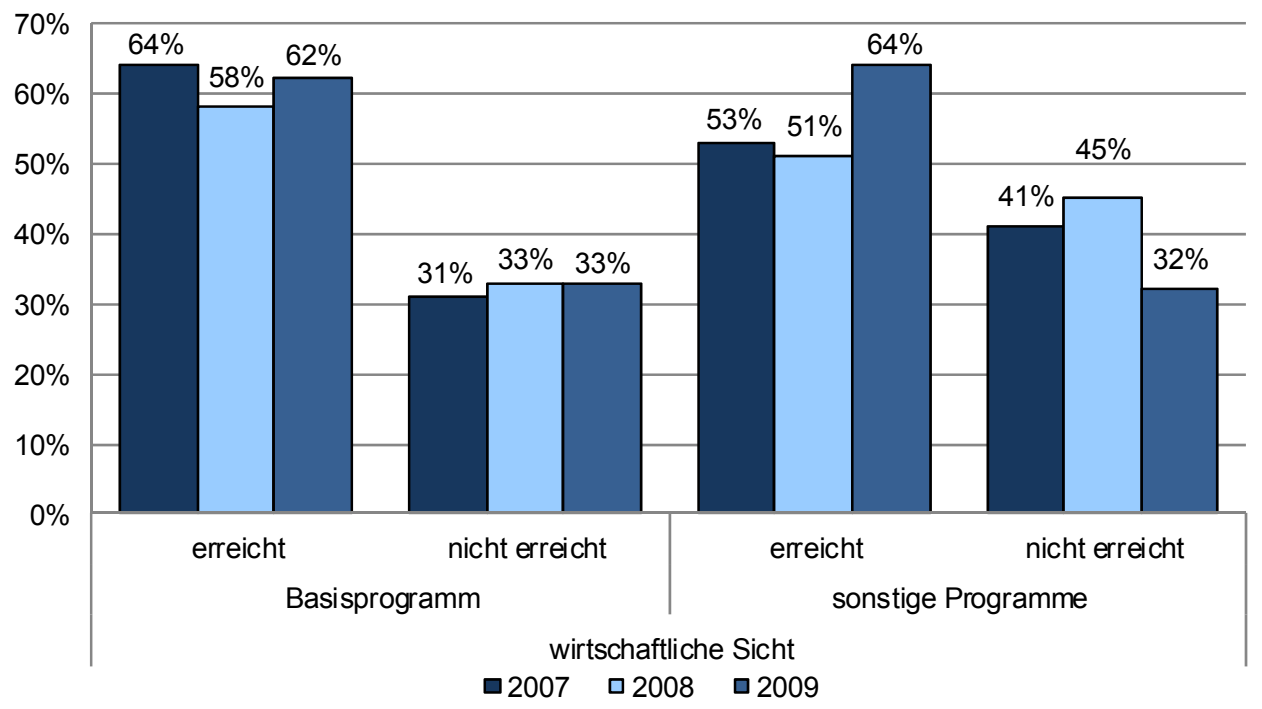

Quelle: KMU Forschung Austria, N = 296, 312, 498. Fehlende Werte auf $100 \%$ = weiß nicht.

Grenzt man die Unternehmen nach der Definition der Europäischen Kommission $a b$, so zeigt sich, dass Kleinunternehmen mit weniger als 50 Mitarbeitern die höchste technische Erfolgsquote aufweisen (91\%). Leicht unter der Gesamterfolgsquote liegen hingegen die mittleren Unternehmen mit $88 \%$. Die zeigt ein identes Muster mit dem Vorjahr. Im Unterschied zum Vorjahr weisen die MU mit knapp zwei Drittel (65\%) jedoch die höchste Erfolgsquote hinsichtlich des wirtschaftlichen Erfolges der FuE-Projekte auf. 
Tabelle 10 Erreichung des Projektzieles aus technischer und wirtschaftlicher Sicht nach Unternehmensgrößenklassen, 2009 abgeschlossene Projekte

\begin{tabular}{|c|c|c|c|c|c|c|c|}
\hline & \multicolumn{2}{|c|}{ KU } & \multicolumn{2}{|c|}{ MU } & \multicolumn{2}{|c|}{ GU } \\
\hline & & Anzahl & $\%$ & Anzahl & $\%$ & Anzahl & $\%$ \\
\hline \multirow{3}{*}{$\begin{array}{l}\text { Projekt- } \\
\text { erfolg aus } \\
\text { technischer } \\
\text { Sicht }\end{array}$} & ja & 130 & $91 \%$ & 114 & $88 \%$ & 183 & $89 \%$ \\
\hline & nein & 13 & $9 \%$ & 15 & $12 \%$ & 22 & $11 \%$ \\
\hline & k.A. & 0 & & 1 & $1 \%$ & 1 & $1 \%$ \\
\hline \multirow{3}{*}{$\begin{array}{l}\text { Projekterfolg } \\
\text { aus wirt- } \\
\text { schaftlicher } \\
\text { Sicht }\end{array}$} & ja & 89 & $62 \%$ & 84 & $65 \%$ & 127 & $62 \%$ \\
\hline & nein & 47 & $33 \%$ & 41 & $32 \%$ & 71 & $35 \%$ \\
\hline & k.A. & 7 & $5 \%$ & 5 & $4 \%$ & 8 & $4 \%$ \\
\hline
\end{tabular}

Quelle: KMU Forschung Austria, N = 498; k.A. = keine Angabe 


\section{Wirtschaftliche Projektergebnisse}

Das Fördervolumen der vorliegenden, und im Jahr 2009 abgeschlossenen 498 Projekte beläuft sich auf rd. $€ 145$ Mio. und die Gesamtkosten der Projekte in den Unternehmen betrugen knapp $€ 274$ Mio. Die Förderquote bezogen auf den Barwert liegt im Mittelwert bei $35 \%$ (Median $30 \%$ ). Der durchschnittliche Förderungsbeitrag (Förderzuschuss, Darlehen und Haftung) liegt bei etwa $€ 291.000$ bzw. der Median bei $€ 165.000$.

Im Vergleich zum Vorjahr verdoppelte sich das gesamte Fördervolumen der betrachteten Projekte (2008: rd. $€ 70$ Mio., 2007: rd. $€ 78$ Mio.), während die Gesamtkosten um rd. $€ 100$ Mio. bzw. 57 \% zulegten. Diese Steigerung ist durch das höhere Volumen verteilter Fördermittel durch die FFG, die Erweiterung der Programme sowie einer in diesem Jahr höheren Rücklaufquote von $77,6 \%$ (siehe Tabellen 1 und 2) begründet.

Die Differenzierung zwischen Basisprogramm und sonstigen Programmen zeigen die unterschiedlichen Volumina auf. Die durchschnittlichen Gesamtkosten eines Projekts der BP beliefen sich auf rd. $€ 648.000$ (Median $€ 353.000$ ), während bei den sonstigen Programmen ein Durchschnittswert von rd. $€ 272.600$ (Median $€$ 83.100) veranschlagt wurde. Des Weiteren liegt die Förderquote bei den Projekten des Basisprogramms bei $23 \%$ (Median), während die sonstigen Programme einen Wert von $50 \%$ aufweisen.

Deutlich unterschiedliche Förderquoten ergibt auch die Differenzierung nach marktnahen und marktfernen Projekten, wobei die als marktfern eingestuften Projekte eine signifikant höhere durchschnittlichen Förderquote aufweisen (60 \% versus $25 \%$ ). Auch bezüglich der Größenklassen lassen sich Unterschiede feststellen. Den höchsten Anteil erhalten KU (Median: $35 \%)$, gefolgt von MU (30\%) und GU (20\%). Es sei jedoch darauf hingewiesen, dass die Gesamtprojektkosten differenziert nach KU, MU und GU ebenfalls variieren, wobei Großunternehmen die finanziell aufwändigsten Forschungsvorhaben durchführen (€ 434.000 im Median). Kleinst- bzw. Kleinunternehmen weisen im Vergleich mediane Gesamtkosten von immerhin $€ 198.000$ auf. 
Tabelle 11 Wirtschaftliche Verwertung der Projektergebnisse der im Jahr 2009 abgeschlossenen Projekte und vergebene Fördermittel

\begin{tabular}{|l|c|c|c|c|}
\hline \multirow{2}{*}{ Die Projektergebnisse ... } & \multicolumn{2}{|c|}{ Projekte } & \multicolumn{2}{c|}{$\begin{array}{c}\text { vergebene Mittel } \\
\text { in Mio. } €\end{array}$} \\
\cline { 2 - 5 } & Anzahl & $\%$ & Anzahl & $\%$ \\
\hline $\begin{array}{l}\text { werden im Unternehmen bereits wirt- } \\
\text { schaftlich verwertet }\end{array}$ & 295 & $59 \%$ & 97,4 & $67 \%$ \\
\hline $\begin{array}{l}\text { werden in Zukunft im Unternehmen wirt- } \\
\text { schaftl. verwertet werden können }\end{array}$ & 43 & $9 \%$ & 16,9 & $12 \%$ \\
\hline $\begin{array}{l}\text { sind im Unternehmen nicht wirtschaftl. } \\
\text { verwertbar, aber sinnvoll für Erkenntniser- } \\
\text { weiterung }\end{array}$ & 114 & $23 \%$ & 20,5 & $14 \%$ \\
\hline $\begin{array}{l}\text { können im Unternehmen nicht verwertet } \\
\text { werden }\end{array}$ & 25 & $5 \%$ & 6,8 & $4 \%$ \\
\hline Keine Angabe & 21 & $4 \%$ & 3,2 & $2 \%$ \\
\hline \hline Gesamt & 498 & $100 \%$ & 145 & $100 \%$ \\
\hline
\end{tabular}

Quelle: KMU Forschung Austria

Von den 2009 abgeschlossenen Projekten konnten $59 \%$ bereits wirtschaftlich verwertet werden und für weitere $9 \%$ wird dies noch erwartet. In zumindest 139 Fällen $(28 \%)$ ist die wirtschaftliche Verwertung der Ergebnisse nicht möglich bzw. wird diese auch in Zukunft nicht erwartet. 23 \%-Punkte der Befragten gaben jedoch an, dass die erzielten Resultate immerhin sinnvoll für die Erkenntniserweiterung waren.

Im Zeitverlauf zeigt sich eine gewisse Volatilität innerhalb eines Korridors, der jedoch die selben Größenverhältnisse beibehält. Während etwa $73 \%$ der abgeschlossenen Projekte im Jahr 2007 bereits einer wirtschaftliche Verwertung zugeführt worden oder dies in Zukunft anvisiert war, waren es $200863 \%$ und 2009 $68 \%$. Nicht verwertbare Projekte lagen innerhalb der Spanne von $24 \%$ (2007) bis $29 \%$ (2008).

Projekte des Basisprogramms können in knapp zwei Drittel der Fälle (65 \%) bereits wirtschaftlich verwertet werden bzw. steht in weiteren $9 \%$ die Kommerzialisierung bevor. Damit haben insgesamt fast drei Viertel $(74 \%)$ der Befragten des Basisprogramms wirtschaftlichen Ertrag in Aussicht gestellt. Innerhalb der sonstigen Programme kann dieser hohe Anteil selbstverständlich nicht erzielt werden. Die Differenz zu den BP liegt bei 23 Prozentpunkten wobei $42 \%$ der Projekte bereits bzw. $9 \%$ künftig verwertet werden können. Als Beitrag zur Erkenntniserweiterung im Unternehmen dienen im Basisprogramm $17 \%$ der abgeschlossenen Projekte, bei den sonstigen Programmen sind es $40 \%$. 
Eine ähnliche Verteilung zeigt die Unterscheidung nach marktnahe/marktferne Projekte. Etwa $73 \%$ der marktnahen Projekte können bereits (63\%) bzw. werden in naher Zukunft (10\%) kommerzialisiert. Gleiches gilt für $40 \%$ bzw. $3 \%$ der marktfernen Projekte. Der größte Anteil der Befragten der marktfernen Projekte gab hingegen an, dass das durchgeführte Projekt zur Erkenntniserweiterung innerhalb des Unternehmens beiträgt (43\%).

Zwei Drittel der aus technischer Sicht erfolgreichen Projekte werden bereits wirtschaftlich verwertet. Von den 295 wirtschaftlich bereits verwerteten Projekte gaben $79 \%$ (233) an, dass sie bereits die damit verbundenen wirtschaftlichen Ziele erreicht haben. Acht Prozent der Projekte weisen Verwertungsaussichten in näherer Zukunft auf. 2 \% (10) der 441 Projekte, die aus technischer Sicht erfolgreich waren, können in keiner Form verwertet werden.

Des Weiteren lässt sich feststellen, dass Großunternehmen besser in der Lage sind, die erzielten Forschungsergebnisse zu verwerten. Insgesamt $65 \%$ der GU haben die Resultate bereits ökonomisch nutzen können, darüber hinaus erwarten sich $9 \%$ kommerzielle Wirkungen in den kommenden Jahren. Bei den KU gilt dies für $59 \%$ bzw. $8 \%$ der Befragten (MU: $58 \%$ bzw. $8 \%$ ). Dementsprechend höher ist der Anteil der erkenntniserweiternden Projekte innerhalb dieser beiden Gruppen (KU und MU jeweils $25 \%$ ) gegenüber den Großunternehmen (20\%).

Die Analyse der wirtschaftlichen Verwertbarkeit der Projektergebnisse in Bezug zu den eingesetzten Mitteln zeigt, dass rd. $67 \%$ des Fördervolumens ( $€ 97,4$ Mio.) in Projekten eingesetzt waren, die bereits wirtschaftlich verwertet werden. $14 \%$ der Gelder (€ 20,5 Mio.) wurden in Projekte investiert, die aus heutiger Sicht zwar wirtschaftlich nicht verwertet werden können, jedoch im Sinne einer Erkenntniserweiterung als sinnvoll betrachtet werden. Bei weiteren rd. $12 \%$ ( $€ 16,9$ Mio.) des Fördervolumens gehen die Unternehmen davon aus, dass in Zukunft eine wirtschaftliche Verwertung möglich sein wird. Die knapp € 6,8 Mio., welche in keiner Weise im Unternehmen verwertet werden können, entsprechen einem relativen Anteil von $7 \%$.

Verglichen mit den Vorjahren ist das vergebene Fördervolumen jener FuEVorhaben, die bereits wirtschaftlich verwertet werden können bzw. künftig kommerzialisiert werden, im aktuellen Untersuchungsjahr am höchsten (2007: 78 \%, 2008: $72 \%$, 2009: $79 \%$ ). Vor allem in Hinblick auf die absoluten Werte ist das Fördervolumen der befragten Projektteilnehmer deutlich höher als in den beiden Vorperioden (wie bereits erwähnt u.a. auf den höheren Rücklauf zurückzuführen). 
Grafik 4 Wirtschaftliche Verwertung der Projektergebnisse der abgeschlossenen Projekte im Zeitverlauf, vergebenes Fördervolumen in Mio. $€$

\begin{tabular}{|c|c|c|c|}
\hline $\begin{array}{r}100 \% \\
90 \%\end{array}$ & 4,5 & 4,4 & 6.8 \\
\hline $90 \%$ & 11,0 & 141 & 20,5 \\
\hline $00 \%$ & 4,4 & & 16,9 \\
\hline $10 \%$ & \multirow{7}{*}{56,3} & 7,2 & \multirow{7}{*}{97,4} \\
\hline $60 \%$ & & \multirow{6}{*}{43,6} & \\
\hline $50 \%$ & & & \\
\hline $40 \%$ & & & \\
\hline $30 \%$ & & & \\
\hline $20 \%$ & & & \\
\hline \multirow[b]{2}{*}{$0 \%$} & & & \\
\hline & 2007 & 2008 & 2009 \\
\hline$\square$ Keine Angabe & 1.900 .000 & 4.400 .000 & 3.200 .000 \\
\hline$\square$ keine Verwertung & 4.500 .000 & 1.300 .000 & 6.800 .000 \\
\hline Erkenntniserweiterung & 11.000 .000 & 14.100 .000 & 20.500 .000 \\
\hline $\begin{array}{c}\text { 口zukünftige wirtschaftl. } \\
\text { Verwertung }\end{array}$ & 4.400 .000 & 7.200 .000 & 16.900 .000 \\
\hline 口 wirtschaftliche Verwertung & 56.300 .000 & 43.600 .000 & 97.400 .000 \\
\hline
\end{tabular}

Quelle: FFG Datenbank und KMU Forschung Austria

Lizenzerlöse, Zusatzumsätze und Umsatzsicherungen stellen eine Einschätzung der direkten Auswirkungen der geförderten Projekte dar. Hier sind natürlich keine Substitutions- oder Multiplikator-Effekte berücksichtigt, wie dies eine Schätzung der gesamtwirtschaftlichen Netto-Effekte benötigen würde. Auch muss in Bezug auf die Interpretation der angeführten Zahlen, der lediglich näherungsweise Charakter der Summen berücksichtigt werden. Wie die nachfolgende Tabelle zeigt, wurde der Großteil dieser Lizenzerlöse, Zusatzumsätze und Umsatzsicherungen im Ausland erzielt, was die traditionell hohe Exportorientierung der geförderten Unternehmen unterstreicht. Zusatzumsätze stellen in diesem Jahr erfreulicherweise die wesentlichere Kategorie dar.

Tabelle 12 Direkte wirtschaftliche Ergebnisse der Befragungsteilnehmer der im Jahr 2009 abgeschlossenen Projekte, in Mio $€$

\begin{tabular}{|c|c|c|}
\hline & $\begin{array}{c}\text { Direktes wirt. } \\
\text { Ergebnis } \\
\text { (in Mio } € \text { ). }\end{array}$ & $\begin{array}{c}\text { davon im Ausland } \\
\text { (in Mio } € \text { ) }\end{array}$ \\
\hline Verwertung der Ergebnisse durch: & 16,4 & $14,5(88 \%)$ \\
\hline Lizenzerlöse & 720,6 & $608,2(84 \%)$ \\
\hline Zusatzumsätze & 536,8 & $435,2(81 \%)$ \\
\hline Umsatzsicherung &
\end{tabular}

Quelle: KMU Forschung Austria, N = 220 (Anzahl jener Befragten, die bei Lizenzerlösen, Zusatzumsätzen oder Umsatzsicherung eine eindeutige Summe angeben konnten) 
Die erwirtschafteten Lizenzerlöse teilen sich auf die Angaben zu 31 Projekten auf, weitere sechs Befragte gaben an Lizenzerlöse zwar erzielt zu haben, konnten oder wollten diese jedoch nicht quantifizieren. Insgesamt 32 der Projekte sind dem Basisprogramm zuzuordnen. Des Weiteren konnten KU am häufigsten in Bezug auf Lizenzerlöse profitieren: 22 Projekte bzw. $60 \%$ der Lizenzierungen gehen auf KU zurück (MU: $19 \%$, GU:19 \%).

114 Teilnehmer meldeten, dass bislang keine Lizenzierung der Projektergebnisse erfolgt ist, obwohl sie das Projektergebnis prinzipiell als lizenzierbar ansehen. Das sind $28 \%$ der technisch erfolgreichen, nicht lizenzierten, Projekte.

Zusätzliche Umsätze konnten im Zuge von insgesamt 216 Projekten (43 \%) erzielt werden, wobei 177 für die Zusammensetzung der obenstehenden Summe verantwortlich zeichnen. Innerhalb des Basisprogramms konnten $50 \%$ der Teilnehmer zusätzliche Umsätze auf Grund der Projektdurchführung generieren, während es bei den sonstigen Programmen $25 \%$ sind. Wie schon bei der Lizenzierung der Projektergebnisse können KU am häufigsten (48 \%) von den Forschungsresultaten profitieren, gefolgt von den MU (45\%) und GU (41\%). Der überwiegende Anteil der Projekte mit Zusatzumsätzen (202 bzw. $94 \%$ ) entstammt den marktnahen Projekten.

Einen Beitrag zur Umsatzsicherung haben 142 der Befragten (rd. 29 \%) identifiziert (90 mit Umsatzangaben). 93 der Projekte trugen sowohl zur Generierung neuer Umsätze als auch zur Umsatzsicherung bei. Beim Basisprogramm hat ein Drittel der untersuchten Projekte zur Sicherung von Unternehmensumsätzen beigetragen (sonstige Projekte: $15 \%$ ). Ähnliche Werte sind bei der Unterscheidung zwischen marktnah und marktfern feststellbar (31\% bzw. $15 \%$ ). In dieser Kategorie konnten ähnliche Anteile der Unternehmensgrößenklassen einen Nutzen generieren (30\% der befragten KU, MU: $29 \%$, GU: $29 \%$ ). 
Grafik 5 Direkte wirtschaftliche Ergebnisse der Befragungsteilnehmer im Zeitverlauf, in Mio $€$

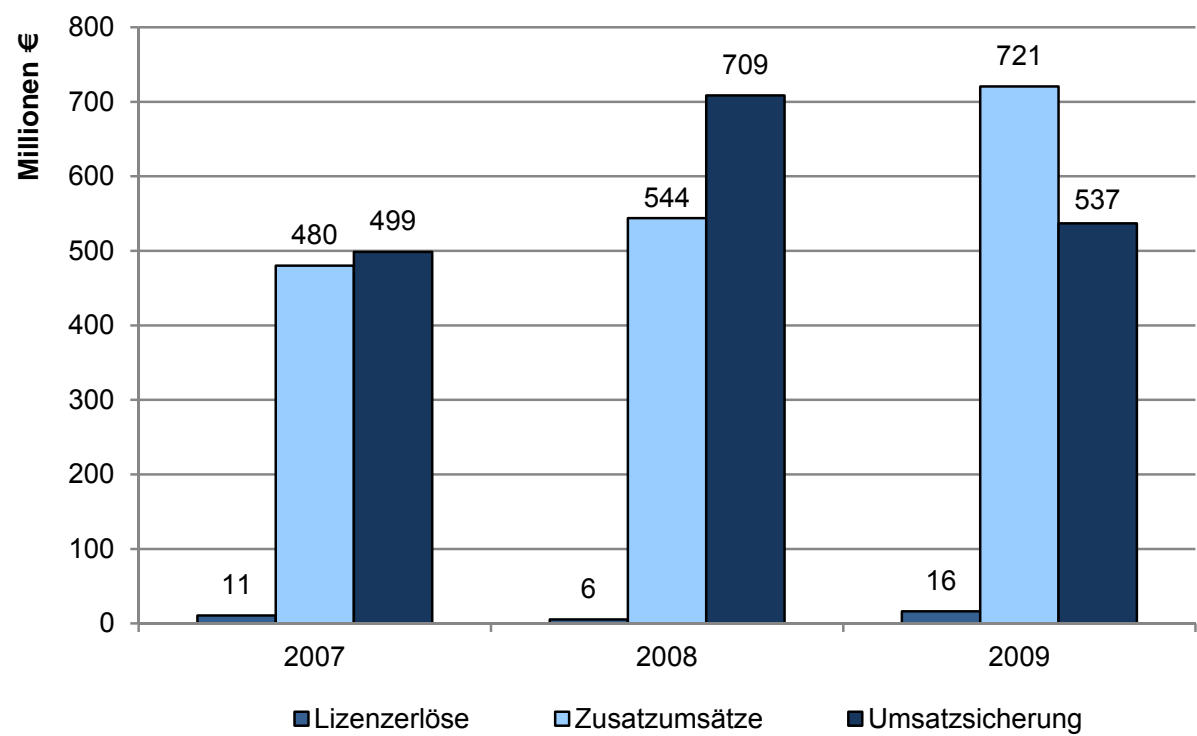

Quelle: KMU Forschung Austria; N = 298

Im Zeitverlauf zeigt sich, dass die Zusatzumsätze inklusive der Umsatzsicherung in den letzten beiden Jahren in Summe beinahe ident ist, wenn auch die Kategorien vertauscht sind. Ebenso wie die Zusatzumsätze zeigen auch die Lizenzerlöse eine Steigerung. Wie auch schon in den Vorjahren wurden die Extremwerte hinsichtlich Plausibilität geprüft, bzw. Ausreißer in der Ermittlung der Ergebnisse bereinigt.

Der Fördermultiplikator stellt eine simple Input/Output Relation der geförderten Projekte dar. Als Maß für den Input wird der Barwert der vergebenen Fördermittel herangezogen. Das Maß für den Output ist die Summe der durch die jeweiligen Projekte realisierten Lizenzerlöse und Zusatzumsätze. Aufgrund der Simplizität dieses Indikators ist weniger die absolute Höhe dessen interessant, sondern eher der relative Unterschied zwischen unterschiedlichen Programmen (mit ihren eigenen Förderlogiken) sowie die Veränderung des Multiplikators über die Zeit.

Bei den im Jahr 2009 abgeschlossenen Projekten beläuft sich der Fördermultiplikator über alle hier abgedeckten FFG Programme auf 9,6. In der Berechnung sind auch marktfernere Projekte der industriellen Forschung enthalten, bei denen vier Jahre nach dem Projektende in der Regel (noch) keine direkten wirtschaftlichen Effekte zu erwarten sind. Weist man den Fördermultiplikator für das Basisprogramm separat aus, dann ergibt sich eine Relation von 1 zu 10,7 (sonstige Programme: 1 zu 4,8). 
Tabelle 13 Fördermultiplikatoren

\begin{tabular}{|c|c|}
\hline Fördermultiplikator gesamt & Fördermultiplikator BP bzw. sonstige P \\
\hline 9,6 & 10,7 bzw. 4,8 \\
\hline Fördermultiplikator marktnahe & Fördermultiplikator marktfern \\
\hline 10,1 & 4,7 \\
\hline
\end{tabular}

Quelle: KMU Forschung Austria; BP = Basisprogramm

Die Unterscheidung zwischen marktnahen sowie marktfernen Projekten resultiert vier Jahre nach Projektende in Multiplikatoren von $1: 10,1$ bzw. $1: 4,7)$. Wie bereits oben erwähnt, sind Projekte im Basisprogramm zur Gänze bei den marktnahen Programmen zugeordnet, sowie ca. die Hälfte der sonstigen Programme.

Insgesamt ist ein etwas niedrigerer Multiplikator der Projekte aus dem Jahr 2009 zu verzeichnen. Mangels einer alternativen Hypothese zur Erklärung dieses Sachverhalts ist es naheliegend, dies unmittelbar mit der sich 2009 entfaltenden Weltwirtschaftskrise in Zusammenhang zu sehen. Der höhere Multiplikator der sonstigen Programme im Vergleich zum Jahr 2008 resultiert daraus, dass die Projekte aus BRIDGE und insbesondere IV2S dieses Jahr höhere wirtschaftliche Erfolge verzeichneten.

\section{Grafik 6 Fördermultiplikatoren der Jahre 2007-2009}

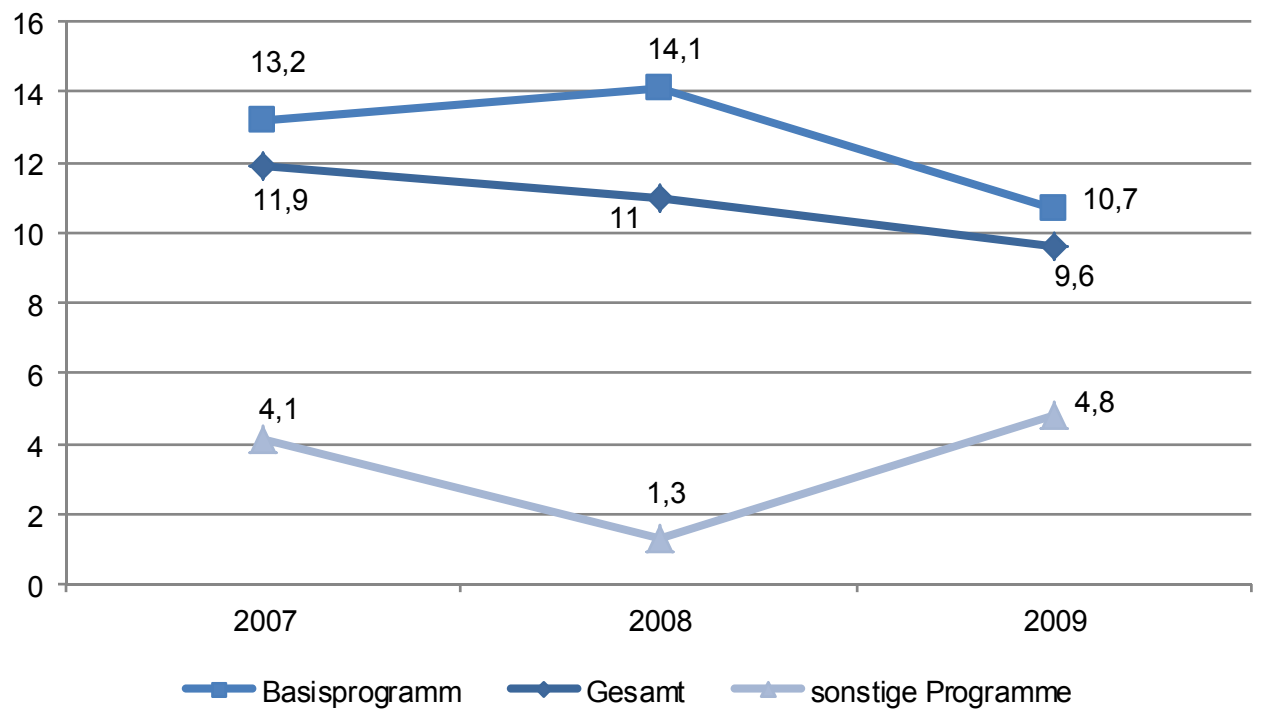

Quelle: KMU Forschung Austria 


\section{Beschäftigungseffekte}

Die im Rahmen des Wirkungsmonitoring untersuchten Projekte zeichneten für einen direkten Beschäftigungseffekt von 1.926 Arbeitsplätzen (nach Köpfen) verantwortlich. Diese Summe ist der Saldo der durch die Projektverwertung gesicherten (739) und neu geschaffenen (1.193) Arbeitsplätze abzüglich der freigesetzten Mitarbeiter/innen (6). ${ }^{4}$ Der Zuwachs der Beschäftigten lässt sich auf die Verwertung von 129 Projekten zurückführen; weitere 16 Befragte gaben an, zusätzliche Mitarbeiter/innen angestellt zu haben, konnten die exakte Zahl jedoch nicht quantifizieren. In 97 Fällen konnte die Aufrechterhaltung des Mitarbeiterstandes gewährleistet werden bzw. haben 32 weitere Fördernehmer angegeben, dass dies ebenfalls auf sie zutreffe, ohne genaue Zahlen anzugeben. In Summe haben 260 Projekte $(52 \%)$ positive Wirkungen auf die Erhaltung bzw. Schaffung von Arbeitsplätzen entfalten können. Die sechs freigesetzten Mitarbeiter/innen teilten sich wiederum auf drei Unternehmen auf. Zusätzlich verneinten 220 Befragte die Frage, ob die Verwertung des Projektes Auswirkungen auf den Beschäftigtenstand ihres Unternehmens hatte; 16 Unternehmen machten keine Angaben.

Die Verteilung der Arbeitsplatzeffekte auf die Unternehmensgröße zeigt, dass GU mit $44 \%$ die häufigsten Fördernehmer - auch am häufigsten zusätzliche Mitarbeiter/innen (55) einstellten oder Arbeitsplätze sicherten (62). Bei den KU stellten 49 Fördernehmer zusätzliche Mitarbeiter/innen an bzw. haben 34 Projekte zur Arbeitsplatzsicherung geführt. In MU nahm die Zahl der Beschäftigten in 41 Fällen $\mathrm{zu}$, weitere $33 \mathrm{mal}$ konnte sie aufgrund des Projektes konstant gehalten werden.

Die Verteilung der Arbeitsplatzeffekte allein nach Beschäftigtengrößenklassen zeigt, dass die untersuchten Projekte in Unternehmen mit mehr als 1.001 Mitarbeiter/innen den größten Anteil am Zuwachs (307) für sich beanspruchen. Bei der Sicherung von Arbeitsplätzen stechen vor allem die Unternehmen mit 501 bis 1.000 Arbeitnehmern (189) heraus. Diese Zahlen sind jedoch mit Vorsicht zu interpretieren, da eine isolierte Betrachtung von Beschäftigungseffekten ohne den dahinterliegenden Eigentümerverhältnissen (insb. international agierende Großunternehmen) nicht ganz verlässlich sein dürfte.

\footnotetext{
${ }^{4}$ Diese Beschäftigungseffekte sind ausschließlich auf die Verwertung der Projekte zurückzuführen. Dies ist im Unterschied zu den Auswirkungen auf die FuE-Mitarbeiterentwicklung während bzw. als Folge der Projektdurchführung, wie weiter hinten beschrieben.
} 
Grafik 7 Beschäftigungseffekte der im Jahr 2009 abgeschlossenen Projekte nach Beschäftigtengrößenklassen der Unternehmen, Anzahl

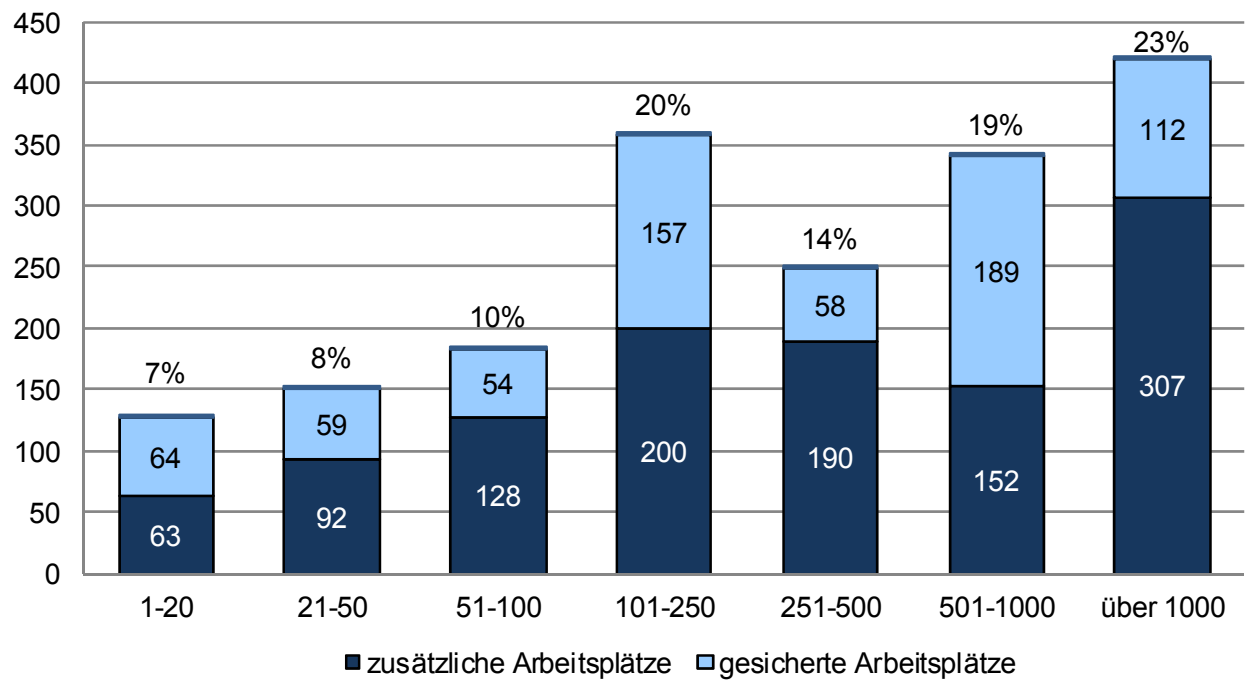

Quelle: KMU Forschung Austria, N = 468

Verglichen mit den Vorperioden konnte im diesjährigen Betrachtungsjahr zwar der absolut höchste Beschäftigungseffekt erzielte werden (2007: 1.723, 2008: 1.513), auf Grund der stark gestiegenen Anzahl untersuchter Projekte ist der relative Zuwachs jedoch eher verhalten geblieben. Ein Grund für die relativ niedrigeren Arbeitsplatzzuwächse könnte im Projektabschlussjahr der FuE-Vorhaben liegen, da diese in die Anfangsphase der weltweiten Wirtschafts- und Finanzkrise fielen.

Der überwiegende Anteil der Projekte mit einer Anstellung neuer Mitarbeiter/innen ist im Basisprogramm zu finden (86\%, 124 Nennungen). Dies entspricht einem Drittel sämtlicher Basisprogramm-Projekte, gegenüber $16 \%$ (21 Projekte) bei den sonstigen Programmen. Hinzu kommen noch 106 bzw. 23 Fälle, in welchen Arbeitsplätze gesichert werden konnten.

Beschäftigungseffekte für FuE-Mitarbeiter/innen (FuE-MA) während bzw. nach der Projektlaufzeit lassen sich bei 255 der 498 Projekte (51\%) feststellen, wobei diese auf externe und interne Rekrutierung zurückzuführen sind. Im Zuge von 180 Projekten (36\%) kam es zu externen Rekrutierungen; zusätzlich gaben 143 Befragte (29\%) an, interne Personalneuzuteilungen getroffen zu haben. Die Zahl der FuEMA, die während der Projektdurchführung extern rekrutiert wurden beläuft sich auf 454, während 335 nach Abschluss eingestellt wurden. Interne Personalrocharden betrafen 198 Mitarbeiter/innen während der Projektdurchführung bzw. 105 Mitarbeiter/innen nach Abschluss des Projekts. 
Der hohe Bedarf an extern rekrutierten bzw. intern zugewiesenen FuE-MA lässt auf eine nicht unwesentlichen Bedarf hochwertiger Arbeitsplätze aufgrund der Projektumsetzung schließen.

Der Anteil der Basisprogrammprojekte, die FuE-Beschäftigte während der Durchführung extern rekrutierten, liegt bei $39 \%$ (404 FuE-MA) bzw. bei $28 \%$ (50) in den sonstigen Programmen. Der Ausbau nach der Projektdurchführung betraf 274 extern rekrutierte FuE-MA im Basisprogramm bzw. 61 in den sonstigen Programmen. Interne Rochaden haben bei 114 BP-Projekten (31 \%) und 29 Projekten (22 \%) der sonstigen Programme stattgefunden. Dabei wurden 160 FuE-MA (BP) während des Projektes zugeteilt bzw. 93 nach Beendigung der FuE-Aktivitäten. Bei den sonstigen Programmen ergeben sich die Personalrochaden zu Werten von 93 (während des Projektes) und 12 (nach Abschluss).

Verglichen nach Größenklassen weisen GU den größten Bedarf an FuE-MA zur Durchführung der geförderten Projekte auf. 316 Mitarbeiter wurden bereits während der Projektdurchführung neu eingestellt bzw. 198 in Folge des Projekts. KU haben 156 Fachkräfte zur Bewältigung des Forschungsaufwandes in die jeweiligen Unternehmen geholt. 76 davon bereits während der Projektdurchführung. Mittlere Unternehmen haben hingegen den geringsten Bedarf gehabt und nur 62 (während des Projektes) bzw. 57 (nach Abschluss) neue FuE-MA aufgenommen. Auch bei internen Rekrutierungstätigkeiten ergibt sich dieses Bild. In GU ist eine Neuzuteilung am häufigsten erfolgt (80 FuE-MA während des Projekts, 46 danach), gefolgt von den KU (61 FuE-MA während des Projekts, 31 danach) und den MU (57 FuEMA während des Projekts, 28 danach).

Die Barwerte der Fördermittel je gesicherten bzw. geschaffenen Arbeitsplatz setzen den Einsatz an Steuermitteln in ein Verhältnis zu etwaigen Arbeitsplatzeffekten. Bei den im Jahr 2009 abgeschlossenen Projekten wurden durchschnittlich rd. $€ 36.600$ an Fördermitteln eingesetzt, um einen Arbeitsplatz zu sichern bzw. zu schaffen. Wie auch in den vergangenen Jahren sind die Unterschiede zwischen Basisprogramm und den sonstigen Programmen hoch, und lassen sich wohl mit den sehr unterschiedlichen Interventionslogiken erklären. Während Basisprogrammprojekte mehrheitlich der experimentellen Entwicklung mit dem unmittelbaren Ziel einer wirtschaftlichen Verwertung zuzurechnen sind, beinhalten die sonstigen Programme ein breitgefächertes Portfolio von Programmen, welche dem Strukturaufbau und thematischen Schwerpunkten zuzurechnen sind. 
Tabelle 14 Barwerte der Fördermittel je gesicherten bzw. geschaffenen Arbeitsplatz durch die im Jahr 2009 abgeschlossenen Projekte, nach Größenklassen in €

\begin{tabular}{|l|c|c|}
\hline \multicolumn{1}{|c|}{2009} & $\begin{array}{c}\text { Barwert der Fördermittel } \\
\text { BP (in } € \text { ) }\end{array}$ & $\begin{array}{c}\text { Barwert der Fördermittel } \\
\text { sonstige Programme (in } € \text { ) }\end{array}$ \\
\hline 1-20 Beschäftigte & 39.550 & 76.220 \\
\hline 21-50 Beschäftigte & 36.350 & 36.550 \\
\hline 51-100 Beschäftigte & 39.800 & 38.240 \\
\hline 101-250 Beschäftigte & 30.000 & 88.120 \\
\hline 251-500 Beschäftigte & 30.120 & 131.010 \\
\hline 501-1.000 Beschäftigte & 28.680 & k.W. \\
\hline über 1.000 Beschäftigte & 28.541 & 95.800 \\
\hline \hline Durchschnitt & 31.660 & 36.500 \\
\hline \hline Durchschnitt gesamt & \multicolumn{2}{|c|}{} \\
\hline
\end{tabular}

Quelle: FFG, KMU Forschung Austria, N = 468; BP = Basisprogramm, gerundet

Die Barwerte liegen teilweise über jenen der Vorjahre (2008: BP rd. $€ 25.000$ und SP $€ 101.500$, bzw. $€ 30.000$ gesamt; 2007: $€ 23.700$ gesamt). Dies ist auf die verhältnismäßig geringeren Beschäftigungseffekte bei gestiegener Zahl der untersuchten Projekte und Fördermittel zurückzuführen. Da sich im Jahr 2009 die Wirtschaftskrise entfaltete, ist ein Zusammenhang mit diesem Ergebnis zu vermuten.

Die obenstehenden Werte je Beschäftigtengrößenklasse sind auf ein Jahr bezogen nicht sehr aussagekräftig, da insbesondere jene der sonstigen Programme großen Schwankungen hinsichtlich der Arbeitsplatzeffekte in den einzelnen Klassen unterliegen. Dieser Effekt kann gemildert werden, indem die Daten von mehreren Jahren in die Berechnung aufgenommen werden. 
Tabelle 15 Durchschnittlicher Barwert der Fördermittel je gesicherten bzw. geschaffenen Arbeitsplatz der in den Jahren 2005 bis 2009 abgeschlossenen Projekte, nach Größenklassen

\begin{tabular}{|l|c|c|c|}
\hline & $\begin{array}{c}\text { Barwert der För- } \\
\text { dermittel BP 2005 - } \\
\text { 2009 (in } € \text { ) }\end{array}$ & $\begin{array}{c}\text { Barwert der För- } \\
\text { dermittel sonstige } \\
\text { Programme 2007 - } \\
\text { 2009 (in } € \text { ) }\end{array}$ & $\begin{array}{c}\text { Barwert der För- } \\
\text { dermittel gesamt } \\
\text { 2005 - 2009 (in } € \text { ) }\end{array}$ \\
\hline 1-20 Beschäftigte & 39.580 & 107.890 & 47.100 \\
\hline 21-50 Beschäftigte & 30.100 & 60.700 & 32.390 \\
\hline 51-100 Beschäftigte & 20.170 & 23.780 & 20.860 \\
\hline 101-250 Beschäftigte & 17.360 & 115.910 & 19.970 \\
\hline 251-500 Beschäftigte & 14.760 & 236.330 & 15.640 \\
\hline 501-1.000 Beschäftigte & 16.100 & 345.080 & 17.345 \\
\hline über 1.000 Beschäftigte & 16.410 & 128.920 & 18.490 \\
\hline \hline Durchschnitt gesamt & 18.720 & 106.280 & 20.790 \\
\hline
\end{tabular}

Quelle: FFG, KMU Forschung Austria; gerundet

Im Durchschnitt der Jahre 2005 bis 2009 wurden rund $€ 21.000$ an Fördermitteln eingesetzt, um einen zusätzlichen Arbeitsplatz zu schaffen bzw. zu sichern. Bei kleineren Unternehmen ist tendenziell ein höheres Fördervolumen je geschaffenen oder gesicherten Arbeitsplatz nötig. Dieses Verhältnis sinkt mit zunehmender Beschäftigtenzahl, erhöht sich aber für Großunternehmen interessanterweise wieder. Der Unterschied zwischen Basisprogramm und sonstigen Programmen ist beträchtlich und ist auf die unterschiedlichen Zielsetzungen der diversen Förderprogramme zurückzuführen. Das Zielsystem so mancher der sonstigen Programme hat nicht primär Arbeitsplatzbeschaffung als Fokus. Darüber hinaus sind die Daten der sonstigen Programme noch mit Vorsicht zu interpretieren, da sie erst seit drei Jahren (2007-2009) in das Monitoring inkludiert werden.

Es sei jedoch darauf hingewiesen, dass es sich auch hier lediglich um Näherungswerte handelt, da die projektbezogenen Wirkungen insbesondere bei Großunternehmen Unschärfen aufweisen. Dies drückt sich unter anderem auch in der Zahl jener Unternehmen aus, die angaben Arbeitsplätze gesichert bzw. geschaffen zu haben, allerdings keine Zahlen nennen konnten. 


\section{Exkurs: Beschäftigtenentwicklung auf Unternehmensebene}

Eine etwas vertiefende Betrachtung der Netto-Beschäftigungswirkung von geförderten Projekten auf das Gesamtunternehmen gibt die unterschiedliche Entwicklung der Beschäftigtenzahl in Unternehmen mit und ohne wirtschaftlichem Projekterfolg. Die zugrunde liegende Frage lautet, welche Beschäftigtenentwicklung die Unternehmen aufweisen, die bis 2013 bereits ihre wirtschaftlichen Ziele mit dem Projekt erzielten, im Vergleich zu jenen Unternehmen, die ihre wirtschaftlichen Projektziele (noch) nicht erreichen konnten.

Das erste Ergebnis der folgenden Grafik ist, dass die Beschäftigtenentwicklung in Unternehmen mit wirtschaftlichem Projekterfolg nicht nur vor dem Projektende (bis 2009) positiv war, sondern sich auch im Zeitraum 2009-13 weiter positiv entwickelte. Während der Median ein starkes Wachstum von 38 \% ausweist, zeigt der Mittelwert ein Halten des hohen Niveaus. Dies führt zum zweiten Ergebnis dieser Darstellung: Da der Median den Einfluss von Großunternehmen auf die Statistik deutlich reduziert, zeigt er, dass kleinere Unternehmen eine dynamischere relative Entwicklung der Beschäftigung verzeichneten als größere Unternehmen. Da kleinere Unternehmen einen niedrigeren Beschäftigtenstand aufweisen, ist dies aufgrund der Arithmetik leicht nachvollziehbar.

Das dritte, durchaus bemerkenswerte Ergebnis ist, dass sich Unternehmen ohne wirtschaftlichem Projekterfolg vor dem Projektende im Jahr 2009 eindeutig positiv entwickelten, dies jedoch nach Projektende nicht fortsetzen konnten. Sowohl der Median als auch der Mittelwert zeigen ein deutlich geringeres Beschäftigungsniveau im Jahr 2013. Hier haben sich kleinere Unternehmen relativ noch negativer entwickelt (Medianentwicklung von $-48 \%$ gegenüber $-21 \%$ im Mittelwert). 
Grafik 8 Entwicklung der Beschäftigtenzahl in Unternehmen mit und ohne wirtschaftlicher Zielerreichung aus den FFG-geförderten Projekten

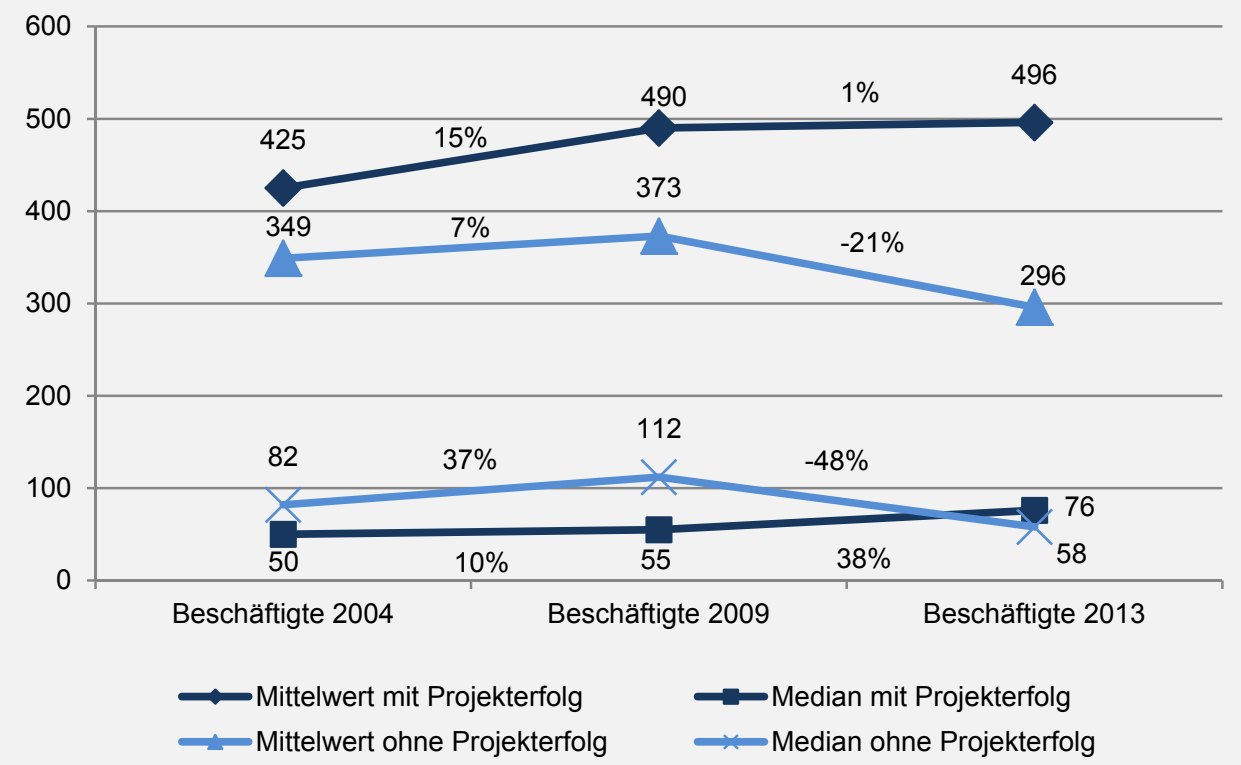

Quelle: FFG Datenbank und KMU Forschung Austria, N = 246 Unternehmen mit wirtschaftlicher Zielerreichung, und 139 Unternehmen ohne wirtschaftlicher Zielerreichung

Der obenstehende Zusammenhang bezüglich der Größenunterschiede wird durch die nachfolgende Grafik noch unterstrichen. Die Darstellung allein für die Unternehmen mit wirtschaftlich erfolgreichen Projekten zeigt, dass KU ihre Mitarbeiterzahl im Zeitraum 2004-13 verdoppelten, MU um $60 \%$ ausweiteten, aber GU ihren Bestand bestenfalls halten konnten. ${ }^{5} \mathrm{KU}$ erfuhren nicht nur vor Projektende im Jahr 2009 die dynamischste Entwicklung, sondern konnten dies nach Projektende noch intensivieren.

Falls man dieser Entwicklung eine kausale Wirkungskette seitens der FFG geförderten Projekte unterstellt, dann würde die nachfolgende Grafik betonen, dass die positiven wirtschaftlichen Impulse aufgrund der Projektergebnisse insbesondere bei $\mathrm{KU}$, aber auch bei $\mathrm{MU}$ in (netto) zusätzliche Beschäftigung münden. GU werden primär jedoch von anderen Einflussfaktoren geleitet. Die obenstehende Analyse der Brutto-Beschäftigungsdaten hat bereits aufgezeigt, dass Projekte von kleineren Unternehmen eher zur Einstellung zusätzlicher Mitarbeiter/innen führen, während Projekte von Großunternehmen - zumindest in den Krisenjahren nach 2008 - eher zur Sicherung von Arbeitsplätzen beitragen.

5 Die Statistik der Sozialversicherung zeigt für die Krisenjahre 2009 und 2010 eine geringere Krisenanfälligkeit für KMU. Der relativ geringere Rückgang des Beschäftigtenstandes in diesen Jahren führte folglich zu einem höheren Anteil an unselbständig Beschäftigten in Österreich (2008: 66,20 \%, 2009: 66,43 \%, 2010: 67,87 \%) im Vergleich zu GU. 
Grafik 9 Entwicklung der Beschäftigtenzahl in Unternehmen mit wirtschaftlicher
Zielerreichung aus den FFG-geförderten Projekten, nach Unternehmensgrößenklassen

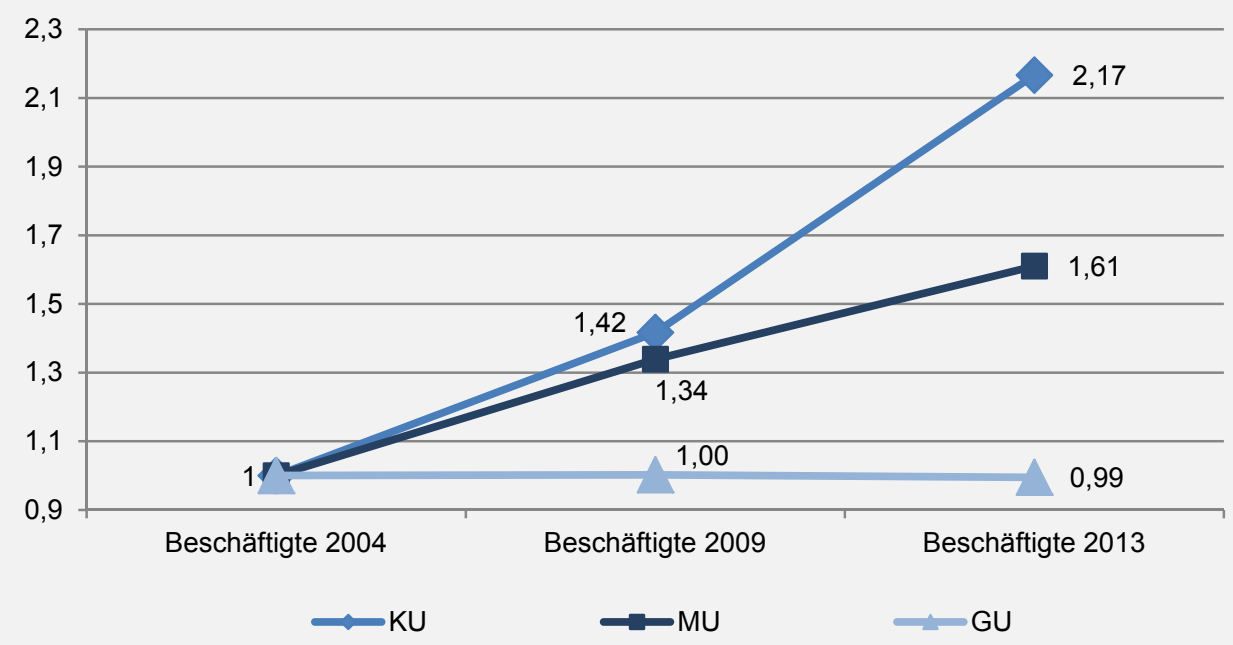

Quelle: FFG Datenbank und KMU Forschung Austria, N = 318 (KU: 91, MU: 87, GU: 132), normalisierte Mittelwerte.

Aus der untenstehenden Grafik 10 lässt sich darüber hinaus ableiten, dass $70 \%$ der ausbezahlten bzw. zugesagten Fördermittel in Projekte geflossen sind, deren wirtschaftliche Projektziele bereits erreicht wurden. Nach Anzahl der Projekte liegt dieser Wert bei $62 \%$ (siehe Grafik 1). ${ }^{6}$

Keine Unterschiede lassen sich hingegen bezüglich der Verteilung der Fördermittel auf die unterschiedlichen Größenklassen feststellen. Jeweils rd. $15 \%$ der verwerteten Förderungen sind den $\mathrm{KU}$ zuzurechnen, rd. $20 \%$ den MU und mehr als die Hälfte $(55 \%)$ den Großunternehmen. Eine größenbedingte Kommerzialisierung der Projektergebnisse ist daher nicht erkennbar. Leichte Abweichungen sind hingegen beim Anteil von Darlehen und Haftungen des Basisprogramms auszumachen, vor allem in der Größenklasse der MU. Bei den Projekten, die ihre wirtschaftlichen Ziele erreichen konnten, liegt der Anteil von Darlehen und Haftungen bei rd. $53 \%$, bei den Projekten ohne wirtschaftlichen Erfolg bei $43 \%{ }^{7}$

${ }^{6}$ Gemäß Grafik 4 erfolgt bereits bei $67 \%$ des Fördervolumens eine wirtschaftliche Verwertung von Projekten. Da die Interviewpartner die wirtschaftlichen Ziele von Projekten mit $70 \%$ des Fördervolumens als erreicht ansahen, folgt daraus, dass einige Projekte kein ökonomisches Interesse verfolgten.

7 Die separate Betrachtung des BP mit und ohne Erreichung der wirtschaftlichen Ziele führt zur selben Aussage. 
Grafik 10 Fördervolumen der abgeschlossenen Projekte mit bzw. ohne wirtschaftlicher Zielerreichung aus den FFG-geförderten Projekten, nach Größenklassen und Form der Zuwendung

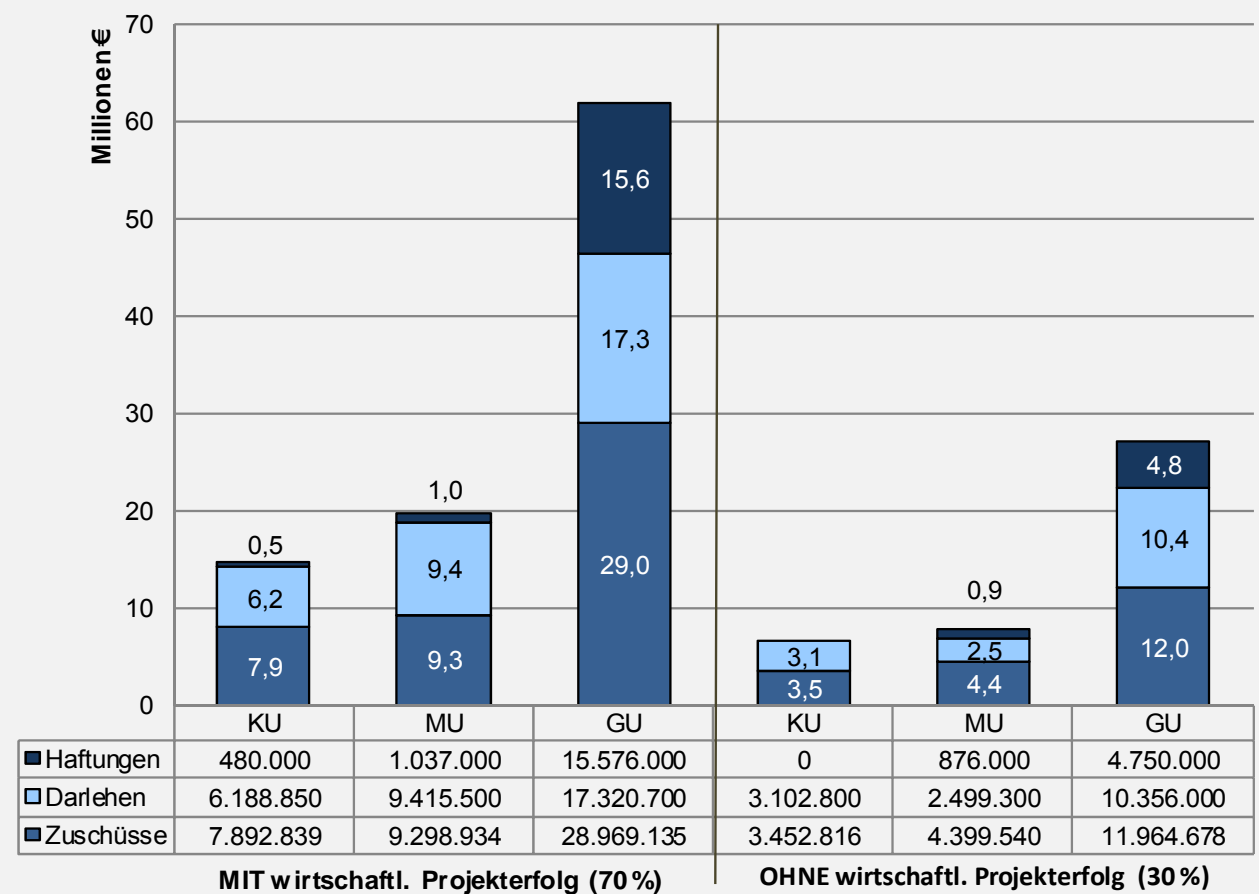

Quelle: KMU Forschung Austria; N = 474 


\section{Auswirkungen auf die Forschungs-, Entwicklungs-, und Innovationsaktivitäten der Unternehmen}

Die im Jahr 2009 abgeschlossenen und im Zuge des Wirkungsmonitorings erhobenen Projekte mündeten in 323 Patente, die das Resultat aus 162 FuE-Vorhaben darstellen. Dies entspricht einem Anteil der Patentneuanmeldungen von $33 \%$ der geförderten Projekte (d. h. bei $40 \%$ der BP-Projekte bzw. durchschnittlich 0,81 Patentanmeldungen je Projekt, und $12 \%$ der sonstigen Programme bzw. 0,17 Patentanmeldungen je Projekt). Als nicht patentierbar wurden die Ergebnisse aus $12 \%$ der Forschungsprojekte (62) eingestuft.

Darüber hinaus wurden die Ergebnisse von 166 Projektteilnahmen wissenschaftlich publiziert (BP: bei $29 \%$ der Projekte bzw. 0,28 Publikationen je Projekt; sonstige Programme: 47 \% der Projekte bzw. 0,47 je Projekt). 61 Projekte der sonstigen Programme wurden auf wissenschaftlicher Ebene veröffentlicht, wobei die BRIDGE-Projekte mit 18 Publikationen ( $r d .30 \%$ ) den größten Anteil ausmachen. Etwas höher liegt der Anteil der marktnahen Projekte, welche sich für 152 der beschriebenen Patentanmeldungen (94\%) und 126 (76\%) der wissenschaftlichen Publikationen verantwortlich zeichnen. Wenig überraschend ist der größte Anteil der Patentmeldungen innerhalb der GU konzentriert: $60 \%$ der Nennungen und 199 aller eingereichten Patente sind in dieser Größenklasse zusammengefasst. Die übrigen Patentanmeldungen teilen sich in etwa zu gleichen Teilen auf KU (60 Patente) und MU (64 Patente) auf. Auch im Bereich der wissenschaftlichen Publikationen liegen GU voran (46\%). Im Vergleich zu den Vorjahren ist der Anteil patentierter Forschungsergebnisse etwa gleichgeblieben (33\% bis $36 \%$ ), wobei die absoluten Zahl der eingetragenen Patente etwas höher liegt (2007: 313, 2008: 198).

9 von 10 der untersuchten Forschungsprojekte (449) führten zu einer Innovation, wovon 444 diese näher bestimmt haben. Wie bereits in den Vorjahren liegt der größte Anteil der Neuerungen im Bereich der Produkte. 250 (56 \%) der Fördernehmer beendeten das Projekt mit einem neuen Produkt (2007: 69 \%, 2008: 60 \%) bzw. führten $26 \%$ der Nennungen in den Bereich veränderte Produkte (2007: $27 \%$, 2008: $27 \%$ ). 126 Projekte führten des Weiteren zu neuen Verfahren (28 \%), womit der Anteil leicht geringer ist als in den beiden Vorjahren (2007: 31 \%, 2008: $29 \%)$. Andere häufig genannte Kategorien sind zudem veränderte Verfahren (20\%), neues Design (12\%) und neue Dienstleistungen (11\%). 
Ein Vergleich zwischen dem Basisprogramm und den sonstigen Programmen zeigt, dass Projekte der BP mehr Produkt- und Verfahrensinnovationen hervorbringen. Sie führten in $54 \%$ der analysierten Projekte zu neuen Produkten (sonstige Programme $39 \%$ ), $27 \%$ gegenüber $21 \%$ entwickelten neue Verfahren und bei $28 \%$ gegenüber $23 \%$ resultierte das Projekt in veränderte Produkte. Andererseits führen Projekte der sonstigen Programme zu einem höheren Anteil von neuen und veränderten Dienstleistungen sowie organisatorischen Innovationen.

Tabelle 16 Arten der Innovation von im Jahr 2009 abgeschlossenen Projekten

\begin{tabular}{|l|c|c|c|c|}
\hline Art der Innovation & Anzahl & $\%$ von N & $\begin{array}{c}\text { Basispro- } \\
\text { gramm }\end{array}$ & $\begin{array}{c}\text { sonstige } \\
\text { Programme }\end{array}$ \\
\hline Neues Produkt & 250 & $56 \%$ & $59 \%$ & $47 \%$ \\
\hline Neues Verfahren & 126 & $28 \%$ & $29 \%$ & $25 \%$ \\
\hline Verändertes Produkt & 117 & $26 \%$ & $28 \%$ & $23 \%$ \\
\hline Verändertes Verfahren & 89 & $20 \%$ & $21 \%$ & $16 \%$ \\
\hline Neues Design für ein Produkt & 53 & $12 \%$ & $13 \%$ & $9 \%$ \\
\hline Neue Dienstleistung & 49 & $11 \%$ & $9 \%$ & $19 \%$ \\
\hline Veränderte Dienstleistung & 27 & $6 \%$ & $5 \%$ & $10 \%$ \\
\hline Organisatorische Innovation & 26 & $6 \%$ & $2 \%$ & $17 \%$ \\
\hline
\end{tabular}

Quelle: KMU Forschung Austria, N = 444, Mehrfachnennungen möglich

Abgesehen von einem leicht niedrigeren Anteil von Dienstleistungen und veränderten Verfahren und Designs zugunsten eines höheren Anteils an neuen Verfahren im Jahr 2009, ist die Verteilung der Innovationsarten über die Jahre bemerkenswert stabil. Die tendenzielle Verringerung von neuen Produkten als Resultat der Projekte ist auf den Anstieg des Anteils der sonstigen Programme im Wirkungsmonitoring zurückzuführen. 


\section{Grafik 11 Arten der Innovation von abgeschlossenen Projekten, 2007-2009}

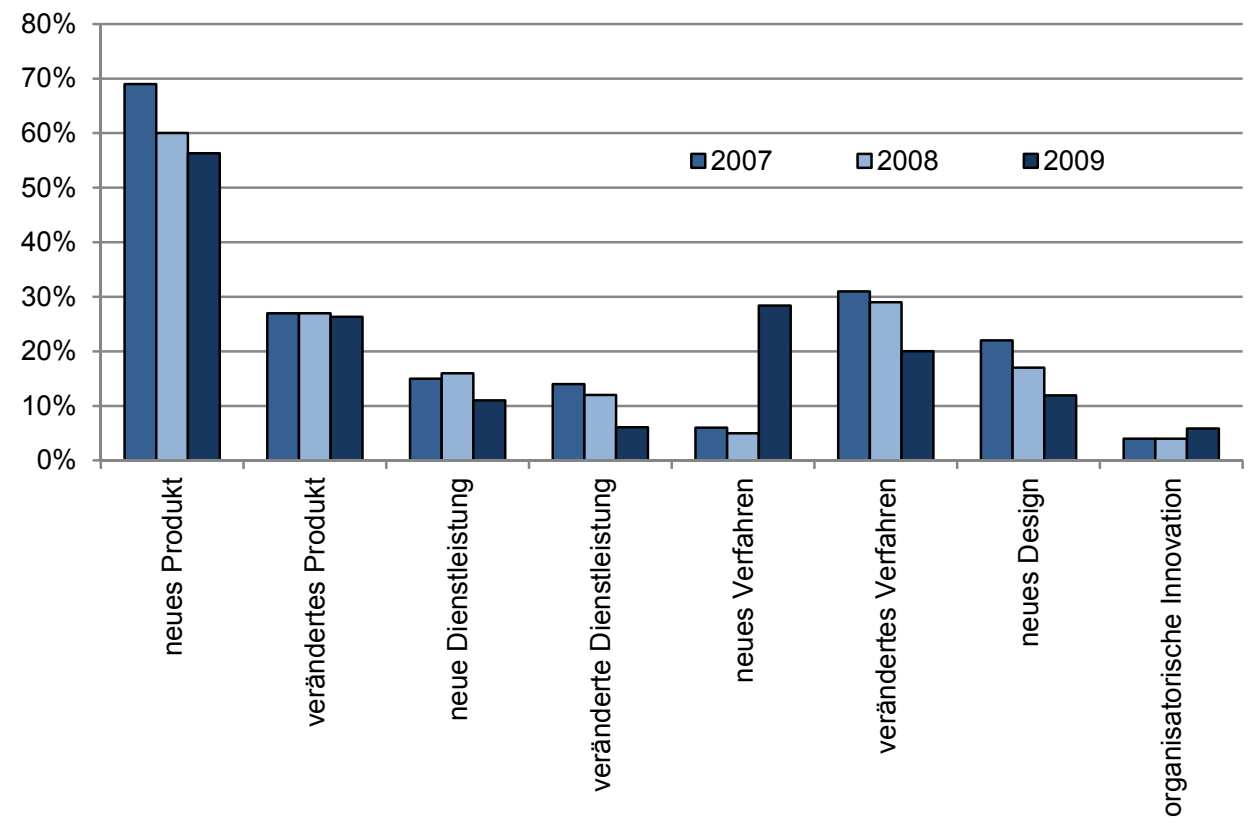

Quelle: KMU Forschung Austria, N für 2007: 276, 2008: 259, 2009: 444, Mehrfachnennungen möglich

In Bezug auf die Unternehmensgrößenklassen lassen sich im Bereich der Produkt und Dienstleistungsinnovationen nur geringe Unterschiede feststellen. Bei den Verfahrensinnovationen betragen die Schwankungen hingegen bis zu $14 \%$ Punkte, wobei $46 \%$ der Großunternehmen und $32 \%$ der KU die Extremwerte darstellen (MU $35 \%$ ).

Die ex-post Einschätzung der Innovationen durch die befragten Unternehmen nach deren Neuheitsgrad ergibt, dass zu einem überwiegenden Teil (66 \%) Neuheiten auf internationaler Ebene erzielt werden konnten (dies impliziert sogleich auch eine nationale Neuheit bzw. eine Firmenneuheit). Bei weiteren $17 \%$ entpuppten sich die Innovationen als eine Neuheit auf dem österreichischen Markt, und in $18 \%$ der Fälle stellen die verwerteten Innovationsergebnisse eine Firmenneuheit dar. Auch in den Vorjahren war die Verteilung sehr ähnlich, wenngleich tendenziell etwas rückläufig (internationale Neuheit 2007: $70 \%$, 2008: $68 \%$ ).

Der überwiegende Anteil (73\%) der befragten Fördernehmer gab an, die Inhalte des Forschungsthemas auch in Folgeprojekten weiterzuverfolgen. Dem gegenüber werden $27 \%$ der Forschungsthemen nicht mehr von den Befragten weiterverfolgt. Damit liegt der Anteil der langfristigen Themenbearbeitung leicht über den Vergleichswerten der Vorjahre (2007: 69 \%, 2008: 69 \%). Von den Projekten des Ba- 
sisprogramms werden $75 \%$ der erzielten Forschungsergebnisse auch in Nachfolgeprojekten weiterverfolgt, während es bei den sonstigen Programmen 68 \% sind.

Eine ähnliche Verteilung lässt sich bei der Unterscheidung zwischen Konsortialprojekten (67\%) und Projekten in alleiniger Durchführung (77\%) erkennen. Den höchsten Anteil der Weiterverfolgung von Forschungsprojekten erzielen die GU, wo $78 \%$ der Projekte auch in weiteren Forschungsthemen Anwendung finden (KU: $68 \%$, MU: $71 \%$ ).

Rund $44 \%$ der Fördernehmer (212) gaben an, in Folge des Forschungsprojektes mindestens eine weitere FuE-Förderung in Anspruch genommen zu haben. Wenig überraschend wurden am häufigsten nationale (148) und regionale (92) Förderprogramme genutzt. Weitere 47 Projekte wurden mit Hilfe europäischer Förderungen durchgeführt. Förderprogramme außerhalb Europas wurden zur Durchführung der untersuchten Projekte nicht bemüht. Wie bei den BP und sonstigen Programmen lassen sich auch zwischen den Unternehmensgrößenklassen nur geringe Unterschiede feststellen. Auch gegenüber den Jahren 2007 (43\%) und 2008 (42\%) lassen sich keine wesentlichen Veränderungen in der Nutzung zusätzlicher Fördergelder feststellen.

Die materielle Infrastruktur wurde im Rahmen von 216 Projekten (43\%) ausgebaut. Bei 184 davon konnten diesbezüglich genaue Angaben getätigt werden. Insgesamt wurden $€ 61,9$ Mio. investiert, durchschnittlich ergibt sich daraus eine Investitionssumme von $€ 336.700$ (Median: $€$ 82.500). Der Anteil der Infrastrukturausgaben an den Gesamtkosten der 184 Projekte lag im Mittel bei rd. $81 \%$, und im Median bei $25 \%$. Die große Differenz ist darin begründet, dass doch einige dieser Unternehmen ein Vielfaches des Projektbudgets in Infrastruktur im Zuge der Produktionsumstellung etc. investierten.

Neben den direkten Wirkungen des Forschungsprojektes auf die Infrastruktur und das Produktportfolio zielt die Analyse auch auf die Wirkungen der geförderten Projekte auf das technologische Niveau, den Know-how Zuwachs, die Kooperationsbereitschaft sowie das Innovationsmanagement des jeweiligen Unternehmens ab.

Bei $93 \%$ der Befragten haben sich demnach das technologische Niveau sowie bei rd. 97 \% der Know-how Zuwachs der Mitarbeiter/innen in fachlich/technischer Sicht zumindest in Teilbereichen verbessert. $45 \%$ konnten einen "insgesamten“ Wissenszuwachs im technologischen Bereich festmachen und $47 \%$ im Bereich des Know-hows. KU profitierten von der Projektteilnahme am stärksten: bei $58 \%$ der Fälle konnten sie eine umfassende Verbesserung feststellen (MU: $47 \%$, GU: $36 \%$ ); $57 \%$ konnten Know-how Zuwächse verzeichnen (MU: $50 \%$, GU: $38 \%$ ), $27 \%$ die Kooperationsbereitschaft verbessern (MU: $28 \%$, GU: $18 \%$ ) und $31 \%$ das Innovationsmanagement effizienter gestalten (MU: $28 \%$, GU: $16 \%$ ). Projekte des Basisprogramms zeigen gegenüber den sonstigen Programmen stärkere Ef- 
fekte. Lediglich im Bereich der Kooperationsbereitschaft ist, wie durch das Programmdesign angestrebt, ein deutlicherer Zugewinn bei den sonstigen Programmen erkennbar (34\% gegenüber $20 \%$ im Basisprogramm). Die Resultate der nachfolgende Tabelle erweisen sich über die Jahre betrachtet als bemerkenswert stabil (nicht abgebildet).

Tabelle 17 Auswirkungen der 2009 abgeschlossenen Projekte auf unterschiedliche Bereiche im Unternehmen, in Prozent

\begin{tabular}{|l|c|c|c|c|}
\hline & $\begin{array}{c}\text { insgesamt } \\
\text { verbessert }\end{array}$ & $\begin{array}{c}\text { in Teilbe- } \\
\text { reichen } \\
\text { verbessert }\end{array}$ & $\begin{array}{c}\text { nicht maß- } \\
\text { geblich } \\
\text { verändert }\end{array}$ & $\begin{array}{c}\text { für das } \\
\text { Projekt } \\
\text { nicht rele- } \\
\text { vant }\end{array}$ \\
\hline $\begin{array}{l}\text { Technologisches Niveau im } \\
\text { Unternehmen }\end{array}$ & $45 \%$ & $48 \%$ & $4 \%$ & $3 \%$ \\
\hline $\begin{array}{l}\text { Know-how Zuwachs der } \\
\text { Mitarbeiter/innen in fachlich/ } \\
\text { technischer Sicht }\end{array}$ & $47 \%$ & $49 \%$ & $3 \%$ & $1 \%$ \\
\hline Kooperationsbereitschaft & $24 \%$ & $40 \%$ & $26 \%$ & $11 \%$ \\
\hline Innovationsmanagement & $24 \%$ & $37 \%$ & $27 \%$ & $12 \%$ \\
\hline
\end{tabular}

Quelle: KMU Forschung Austria, N = 494 (Zeile 1), 493 (Z 2), 486 (Z 3), 485 (Z 4)

In 269 Projekten (55\%) konnten hinsichtlich einer stärkeren Integration in FuEKooperationen Fortschritte erzielt werden. Der größte Anteil davon ist auf europäischer Ebene zu verzeichnen (29\%), gefolgt von nationalen Innovationskooperationen $(26 \%)$. Nach Programmtypen profitieren die sonstigen Programme stärker auf regionaler (17\%) und nationaler (39\%) Ebene (BP: $12 \%$ bzw. $21 \%$ ), während das Basisprogramm stärker auf europäischer $(29 \%)$ und internationaler Ebene (11\%) wirkt (sonstige Programme: $25 \%$ bzw. $9 \%$ ). Gegenüber den Vorjahren lassen sich sowohl Zuwächse (regional 2007: $10 \%$, 2008: $7 \%$ ), konstante Werte (Europa 2007: $28 \%, 2008: 30 \%$ ) als auch rückläufige Kooperationszuwächse (außerhalb Europas 2007: 12 \%, 2008: $13 \%$ ) feststellen. Nach Unternehmensgrößenklassen weisen die MU geringere Integration in Innovationskooperationen auf (trifft nicht zu KU: 14 \%, MU: $27 \%$, GU: $15 \%$ ). 
Tabelle 18 Auswirkungen auf Kooperationen und Märkte, 2009 abgeschlossen

\begin{tabular}{|l|c|c|c|c|c|c|}
\hline & regional & national & $\begin{array}{c}\text { in } \\
\text { Europa }\end{array}$ & $\begin{array}{c}\text { außer- } \\
\text { halb } \\
\text { Europa }\end{array}$ & $\begin{array}{c}\text { war } \\
\text { nicht } \\
\text { geplant }\end{array}$ & $\begin{array}{c}\text { trifft } \\
\text { nicht zu }\end{array}$ \\
\hline $\begin{array}{l}\text { Mein Unternehmen ist } \\
\text { durch das Projekt stärker } \\
\text { in FuE- und Innovations- } \\
\text { kooperationen inte- } \\
\text { griert... *) }\end{array}$ & $13 \%$ & $26 \%$ & $29 \%$ & $10 \%$ & $29 \%$ & $18 \%$ \\
\hline $\begin{array}{l}\text { Mein Unternehmen konn- } \\
\text { te durch das Projekt neue } \\
\text { Märkte erschließen...** }\end{array}$ & $6 \%$ & $15 \%$ & $37 \%$ & $25 \%$ & $20 \%$ & $27 \%$ \\
\hline
\end{tabular}

Quelle: KMU Forschung Austria, $\left.\left.{ }^{*}\right) \mathrm{N}=489,{ }^{* *}\right) \mathrm{N}=484$, Mehrfachnennungen möglich

Die Erschließung neuer Märkte aufgrund der Projektdurchführung gelang 260 der befragten Fördernehmer (54\%). Im Zentrum der Markterschließungen stand hierbei die europäische Orientierung (37\%) sowie Märkte außerhalb Europas (27\%). Die internationale Ausrichtung der Innovationstätigkeiten ist vor allem dem Basisprogramm zuzurechnen, von welchen rd. $42 \%$ bzw. $31 \%$ europäische bzw. internationale Märkte erschließen konnten. Nur $19 \%$ der Projektträger der sonstigen Programme haben neue, europäische Märkte erschlossen bzw. $7 \%$ auch außerhalb Europas Fuß gefasst. Die geförderten Projekte sind für $\mathrm{KU}$ am häufigsten der Anstoß zur verstärkten Internationalisierung (Europa: $41 \%$ bzw. $27 \%$ außerhalb), während MU und GU bereits präsenter sind (MU: $35 \%$ bzw. $22 \%$; GU: $32 \%$ und $24 \%$ ). Gegenüber den Vorjahren war die Markterschließung rückläufig bzw. konstant.

Die Fähigkeit von Unternehmen, neue Technologien zu absorbieren und zu implementieren, hängt in starkem Maße von ihrer Kooperationstätigkeit ab. In den neueren Innovationstheorien wird diese sog. "absorptive capacity" zunehmend auch als ein wesentlicher Bestandteil der Wirkungen von Förderungen betrachtet.

Die Forschungsaktivitäten der befragten Unternehmen haben dazu beigetragen, dass knapp $60 \%$ der Projekte zu neuen Kontakten geführt haben (2007: $51 \%$, 2008: $57 \%$ ). Des Weiteren sind in Folge von etwa $64 \%$ bestehende Kontakte intensiviert worden (2007: $58 \%$, 2008: $57 \%$ ). Die neuen und intensivierten Kontakte wurden 271 Fällen (55\%) auch in Folgeprojekten weitergeführt. Des Weiteren sind rd. $11 \%$ der Kontakte in gemeinsamen wissenschaftlichen Publikationen weitergeführt worden bzw. sind $28 \%$ ohne konkrete Folgeprojekte erhalten geblieben. Nach lediglich 44 Projektabschlüssen ist in der Folge auf weitere Zusammenarbeit verzichtet worden. BP und sonstige Programme weisen ähnliche Werte auf, größere Schwankungen sind lediglich in der Weiterführung im Zuge wissenschaftlicher Publikationen feststellbar (BP: 8 \%; sonstige Programme: $17 \%$ ). 
Die untenstehende Tabelle zeigt in welchen Bereichen neue Kontakte geschlossen bzw. die bereits vorhandenen Kontakte intensiviert wurden. Im Gegensatz zu den beiden Vorjahren wurden die Universitäten als wichtigste, neue Kontakte im Inland von den Zulieferern und den Abnehmern abgelöst. Dies unterstreicht einmal mehr die hohe Relevanz der Wertschöpfungsketten im Innovationsprozess, und andererseits einen möglichen Bei den bestehenden Kontakten im Inland konnte diese Spitzenposition hingegen gehalten werden. Leicht zugenommen hat hingegen die Kontaktschließung mit Fachhochschulen im In- und Ausland (2007 $10 \%$; 2008: $12 \%$; 2009: 14\%).

Tabelle 19 Kontakte nach Bereichen, 2009 abgeschlossen

\begin{tabular}{|l|c|c|c|c|}
\hline \multirow{2}{*}{} & \multicolumn{2}{|c|}{ Inland } & \multicolumn{2}{c|}{ Ausland } \\
\cline { 2 - 5 } & $\begin{array}{c}\text { neue } \\
\text { Kontakte }\end{array}$ & $\begin{array}{c}\text { bestehende } \\
\text { Kontakte }\end{array}$ & $\begin{array}{c}\text { neue } \\
\text { Kontakte }\end{array}$ & $\begin{array}{c}\text { bestehende } \\
\text { Kontakte }\end{array}$ \\
\hline Universitäten & $24 \%$ & $38 \%$ & $12 \%$ & $7 \%$ \\
\hline Fachhochschulen & $14 \%$ & $14 \%$ & $4 \%$ & $1 \%$ \\
\hline $\begin{array}{l}\text { Außeruniversitäre Forschungs- } \\
\text { einrichtungen }\end{array}$ & $15 \%$ & $23 \%$ & $10 \%$ & $5 \%$ \\
\hline Andere Unternehmen, und zwar... & $6 \%$ & $15 \%$ & $4 \%$ & $10 \%$ \\
\hline $\begin{array}{l}\text { Innerhalb der Unternehmens- } \\
\text { gruppe }\end{array}$ & $28 \%$ & $22 \%$ & $20 \%$ & $12 \%$ \\
\hline (Potenzielle) Zulieferer & $28 \%$ & $24 \%$ & $29 \%$ & $18 \%$ \\
\hline (Potenzielle) Abnehmer & $5 \%$ & $2 \%$ & $9 \%$ & $6 \%$ \\
\hline (Potenzielle) Konkurrenten & $1 \%$ & $1 \%$ & $2 \%$ & $1 \%$ \\
\hline Sonstiges & & & \\
\hline
\end{tabular}

Quelle: KMU Forschung Austria, N = 414

Während Projekte des Basisprogramms deutlich häufiger Kontakte mit anderen Unternehmen suchen bzw. intensivieren, sind die sonstigen Programme stärker mit inländischen Partnern aus der Wissenschaft verknüpft. 


\section{Exkurs: Neukunden versus Bestandskunden}

Als Neukunden werden Unternehmen definiert, die vor dem Jahr 2005 kein Projekt bei der FFG erfolgreich abgeschlossen haben. Insgesamt wurden im Zuge der diesjährigen Befragung 171 Projekte von Neukunden (34\%) bzw. 327 von Bestandskunden durchgeführt (66\%). Von den Neukunden wurden fast drei Viertel der Projekte (73\%) im Rahmen des Basisprogramms durchgeführt und $27 \%$ im Zuge der sonstigen Programme.

Betrachtet man die Verteilung auf Unternehmensebene, so sind $40 \%$ (162) der befragten Unternehmen Neukunden und $60 \%$ (244) bereits Bestehende. Rund die Hälfte der Neukunden (51\%) sind der Größenklasse der KU zuzurechnen, 31 \% sind MU und nur $19 \%$ GU. Umgekehrt sind $48 \%$ der Bestandskunden GU und nur $23 \%$ Kleinst- und Kleinunternehmen. Für knapp $20 \%$ der Neukunden-Projekte war das Projekt die erste FuE-Aktivität. Etwa $77 \%$ haben hingegen ihre FuEAktivitäten ausgebaut. Sowohl $60 \%$ der Bestands- als auch der Neukunden haben die Projekte in Eigenregie durchgeführt.

Die technische und wirtschaftliche Zielerreichung zeigt Abweichungen von jeweils vier Prozentpunkten - technischer Erfolg Neukunden: $86 \%$ bzw. $90 \%$ bei Bestandskunden; wirtschaftlicher Erfolg Neukunden: $60 \%$ bzw. $64 \%$ bei Bestandskunden.

Etwas größere Unterschiede gibt es bei der wirtschaftlichen Verwertung im Zuge von 90 Projekten (53\%) der Neukunden (Bestandskunden 205 bzw. 63 \%). Weitere $11 \%$ der neuen FFG Kunden erwarten sich die wirtschaftliche Verwertbarkeit in den kommenden Jahren (Bestandskunden: 8\%). Zur Erkenntniserweiterung dienten $28 \%$ der durchgeführten Projekte bei den Neukunden und $20 \%$ bei den Bestandskunden.

Auswirkungen der Projektverwertung auf die Beschäftigung sind häufiger bei den bestehenden Kunden festzustellen. $29 \%$ der Projekte haben zu neuen Mitarbeitern geführt und in $28 \%$ der Fälle konnten Arbeitsplätze gesichert werden. Bei den Projekten der Neukunden waren hingegen 32 \% (neu) bzw. $21 \%$ (gesichert) dazu imstande.

Weitere Förderungen wurden sowohl von $43 \%$ der Bestandskunden als auch der Neukunden in Anspruch genommen. Während Bestandskunden häufiger regionale Förderprogramme in Anspruch nahmen (19 \% vs. $10 \%$ ), weisen Neukunden einen nahezu äquivalenten Anteil auf nationaler Ebene aus (30\% vs. $28 \%$ ). 
Die Entstehung neuer Kontakte ist in beiden Kundengruppen etwa gleich stark ausgeprägt und liegt bei $54 \%$ (Neukunden) und $56 \%$ (Bestandskunden). Bei der Weiterführung der Kontakte nach Projektabschluss ist der Anteil bei den Bestandskunden etwas geringer (60\%) als bei den Neukunden (69\%). Die Additionalitätsfrage ergibt, dass $29 \%$ der Neukunden dieses Projekt ohne Förderung in keiner Form hätten umsetzen können (Bestandskunden $20 \%$ ), was auch den Ergebnissen der letzten Jahre entspricht.

Insgesamt lässt sich festhalten, dass Neukunden für die FFG einen relativ höheren Nutzen hinsichtlich der "weichen" Innovationsfaktoren aus der FFG Forschung ableiten, aber hinsichtlich der kommerziellen Verwertung der Forschungsergebnisse hinter den Bestandskunden liegen, die bereits mehr Erfahrung mit derartigen Projekten aufweisen. 


\section{Additionalität}

Die Frage nach der Additionalität der Förderung gibt Auskunft darüber, in welchem Ausmaß das entsprechende Projekt von den Unternehmen auch ohne Erhalt der Förderung durchgeführt worden wäre. $23 \%$ der Befragten (116) gaben an, dass eine Projektdurchführung ohne Fördermittel nicht möglich gewesen wäre. Von diesen Unternehmen gaben 103 in einer offenen Fragestellung auch eine Begründung dafür. Zwei Drittel wären nicht in der Lage gewesen, die notwendigen finanzielle Mittel zur Verfügung zu stellen; $21 \%$ hätten das Projektrisiko als zu hoch erachtet und weitere $12 \%$ der Befragten nannten andere Gründe. Darunter etwa der notwendige Kooperationsbedarf für das Projekt oder eine damals im Raum gestandene Verlagerung der Forschungsaktivitäten in das Ausland. Der Vergleich zwischen Basisprogramm (16\%) und sonstigen Programmen (44\%) zeigt, dass ein deutlich höherer Anteil der Projektteilnehmer der sonstigen Programme das FuE-Projekt ohne Förderung nicht durchführen hätte können.

In $4 \%$ der betrachteten Fälle kommt zu einem vollständigen Crowding-out privater Innovationsausgaben durch die Förderung des Forschungsprojekts. Die betroffenen 21 FuE-Vorhaben wären auch ohne Zuhilfenahme externer Fördermittel in unverändertem Ausmaß durchgeführt worden. Weitere $15 \%$ der untersuchten Forschungsprojekte wären zumindest in überwiegendem Ausmaß vorangetrieben worden. Der überwiegende Teil der geförderten FuE-Vorhaben, nämlich 286 Projekte bzw. 58 \%, hätte im Falle ausbleibender Förderungen die angestrebten Innovationsbemühungen in „deutlich geringerem“ Ausmaß durchführen können.

Bei $85 \%$ der Befragten, die das Projekt ohne Förderung durchgeführt hätten bzw. in überwiegendem oder deutlich geringerem Ausmaß, wäre das Projekt zeitlich verzögert umgesetzt worden. Darüber hinaus haben $16 \%$ angegeben, dass die Projektumsetzung ohne Kooperationspartner durchgeführt worden wäre, obwohl dies so geplant war. In rd. $28 \%$ der Projekte war die Umsetzung ohnehin ohne Kooperationspartner geplant. 
Grafik 12 Hätten Sie das Projekt auch ohne Förderung durchgeführt? Jahr 2009

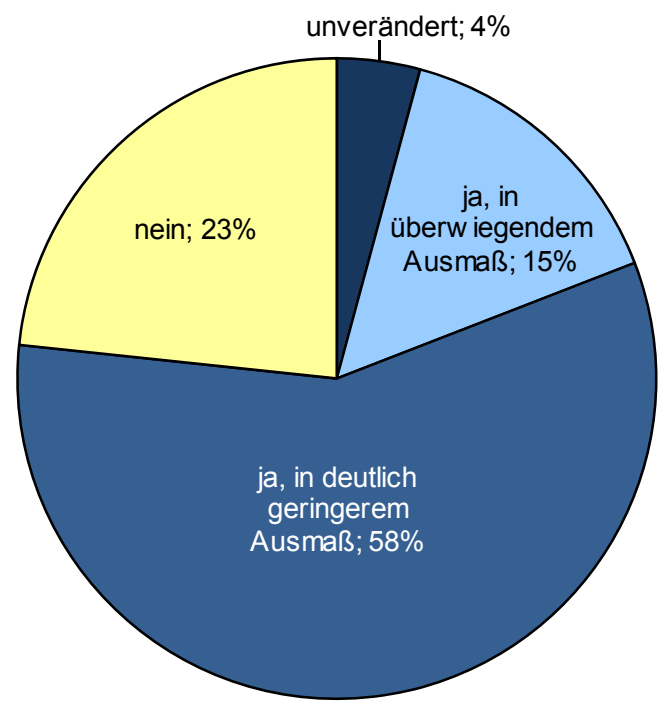

w enn ja, dann zeitlich verzögerte Umsetzung: $85 \%$ w enn ja, dann Umsetzung ohne Kooperationspartner: $16 \%$

Quelle: KMU Forschung Austria, N = 497

Im Vergleich zu den Vorjahren sind diese Werte bemerkenswert konstant. Die deutlichste Schwankung ist in der Gruppe jener Projekte feststellbar, die ohne Förderung nur in geringerem Ausmaß durchgeführt worden wäre (Schwankungsbreite fünf Prozentpunkte). Im Verhältnis zu den Vorjahren kann des Weiteren eine höhere Zielorientierung der eingesetzten Fördermittel festgestellt werden. Während 2007 ein Fünftel der geförderten Projekte nicht durchgeführt worden wären, sind es in den 2008 und 2009 bereits $23 \%$. 
Grafik 13 Hätten Sie das Projekt auch ohne Förderung durchgeführt? Abgeschlossene Projekte der Jahre 2007-2009

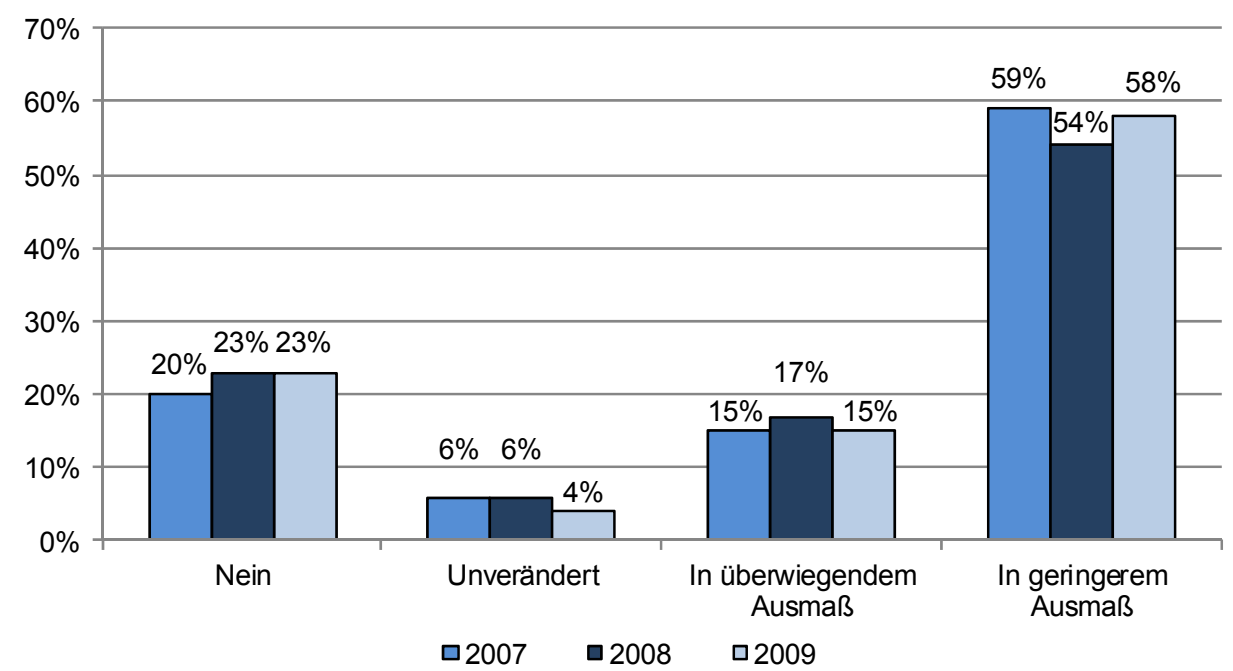

Quelle: KMU Forschung Austria

Die Unterscheidung zwischen Basisprogramm und sonstigen Programmen zeigt auf, dass Projekte der letzteren Kategorie überproportional durch die Förderung stimuliert werden. $44 \%$ der Projekte der sonstigen Programme wären ohne die entsprechenden Fördermittel nicht durchgeführt worden. Dementsprechend geringer ist der Anteil im Basisprogramm (16\%); hier wäre die überwiegende Mehrheit der Projekte $(61 \%)$ in deutlich niedrigerem Ausmaß durchgeführt worden. Differenziert zwischen marktnahen und marktfernen Projekten zeigen sich ebenfalls deutliche Unterschiede in der Projektdurchführung. Wenig überraschend weisen Projekte mit marktfernem Charakter eine höhere Abhängigkeit von Fördermitteln auf (nein: $42 \%$ ) als marktnahe Projekte (nein: $20 \%$ ). Weitere $50 \%$ markferner FuE-Vorhaben wären nur in einem deutlich reduzierten Rahmen durchgeführt worden (marktnahe $59 \%$ ).

Hinsichtlich der unterschiedlichen Unternehmensgrößen zeigen sich erwartbare Muster: je kleiner das Unternehmen, desto geringer die Bereitschaft der Projektdurchführung ohne Fördermittel (Kleinstunternehmen: $45 \%$, KU $28 \%$, MU $15 \%$, GU $14 \%$ ). Erwähnenswert ist des Weiteren, dass MU und GU ihre Projekte zu $6 \%$ bzw. 7\% unverändert durchgeführt hätten. Kleinst- und Kleinunternehmen hätten dies nur in $1 \%$ bzw. $2 \%$ der untersuchten Fälle zustande gebracht.

Zwischen erstgeförderten Unternehmen und Bestandskunden der FFG sind Differenzen in geringerem Ausmaß feststellbar. Projekte die von "neuen“ Fördernehmern abgewickelt werden, hätten in $29 \%$ der Fälle ohne Förderung nicht stattgefunden. Bestandskunden weisen einen Wert von $20 \%$ auf. 


\section{Anhang}

\section{Berechnungsformeln}

In der Folge werden die Berechnungsformeln zu den Auswertungen angeführt.

\section{Beschäftigungseffekte}

zusätzliche Mitarbeiter/innen

+ gesicherte Arbeitsplätze

- $\quad$ freigesetzte Mitarbeiter/innen

Gesamtbeschäftigungseffekt

\section{Multiplikator}

$$
\text { Fördermultiplikator M3 }=\frac{\text { Lizenzerlöse + Zusatzumsätze }}{\text { Barwert der Förderungen }}
$$




\section{Tabellen}

Tabelle 20 KMU-Definition der Europäische Kommission

\begin{tabular}{|l|c|c|c|}
\hline Unternehmenskategorie & Mitarbeiterlinnen & Umsatz & Bilanzsumme \\
\hline Kleinstunternehmen & $<10$ & $\leq 2$ Mio. EUR & $\leq 2$ Mio. EUR \\
\hline Kleinunternehmen & $<50$ & $\leq 10$ Mio. EUR & $\leq 10$ Mio. EUR \\
\hline Mittlere Unternehmen & $<250$ & $\leq 50$ Mio. EUR & $\leq 43$ Mio. EUR \\
\hline Großunternehmen & \multicolumn{3}{|c|}{ Abweichende Werte bzw. Eigentümerverhältnisse } \\
\hline
\end{tabular}

Quelle: Europäische Kommission

Diese Grenzwerte dürfen auch gemeinsam mit „Partnerunternehmen“ bzw. „Verbundenen Unternehmen“ in Summe nicht überschritten werden. 
Tabelle 21 Frage 8: Wurde das Projektziel aus technisch / wirtschaftlicher Sicht erreicht? Im Bundesländervergleich

\begin{tabular}{|c|c|c|c|c|c|c|c|c|c|c|c|c|c|c|c|c|c|c|c|c|}
\hline & \multicolumn{2}{|c|}{ Gesamt } & \multicolumn{2}{|c|}{ Burgenland } & \multicolumn{2}{|c|}{ Kärnten } & \multicolumn{2}{|c|}{$\begin{array}{l}\text { Niederöster- } \\
\text { reich }\end{array}$} & \multicolumn{2}{|c|}{$\begin{array}{l}\text { Oberöster- } \\
\text { reich }\end{array}$} & \multicolumn{2}{|c|}{ Salzburg } & \multicolumn{2}{|c|}{ Steiermark } & \multicolumn{2}{|c|}{ Tirol } & \multicolumn{2}{|c|}{ Vorarlberg } & \multicolumn{2}{|c|}{ Wien } \\
\hline & abs.. & in $\%$ & abs. & in $\%$ & abs. & in $\%$ & abs. & in $\%$ & abs. & in $\%$ & abs. & in $\%$ & abs. & in $\%$ & abs. & in $\%$ & abs. & in $\%$ & abs. & in $\%$ \\
\hline Projekte insgesamt & 498 & 100,0 & 8 & 1,6 & 27 & 5,4 & 52 & 10,4 & 112 & 22,5 & 26 & 5,2 & 115 & 23,1 & 34 & 6,8 & 32 & 6,4 & 92 & 18,5 \\
\hline Förderungsbeiträge *) & 69,4 & 100,0 & 1,1 & 1,6 & 3,2 & 4,6 & 4,9 & 7,1 & 16,4 & 23,6 & 3,8 & 5,4 & 21,1 & 30,4 & 5,2 & 7,5 & 3,8 & 5,5 & 10,0 & 14,4 \\
\hline Darlehen *) & 50,3 & 100,0 & 0,9 & 1,8 & 3,0 & 6,0 & 4,1 & 8,2 & 10,3 & 20,4 & 3,1 & 6,2 & 14,1 & 28,0 & 4,1 & 8,1 & 4,1 & 8,1 & 6,7 & 13,3 \\
\hline technisch erreicht & 441 & 88,6 & 6 & 1,4 & 23 & 5,2 & 43 & 9,8 & 98 & 22,2 & 20 & 4,5 & 102 & 23,1 & 32 & 7,3 & 30 & 6,8 & 87 & 19,7 \\
\hline Förderungsbeiträge *) & 62,6 & 90,2 & 0,3 & 0,5 & 2,9 & 4,6 & 4,1 & 6,5 & 15,0 & 24,0 & 3,0 & 4,9 & 18,9 & 30,2 & 5,1 & 8,1 & 3,6 & 5,7 & 9,6 & 15,4 \\
\hline Darlehen *) & 45,3 & 90,0 & 0,6 & 1,4 & 2,5 & 5,5 & 3,4 & 7,4 & 9,0 & 19,9 & 2,7 & 5,9 & 12,9 & 28,4 & 3,9 & 8,7 & 3,8 & 8,5 & 6,4 & 14,2 \\
\hline $\begin{array}{l}\text { wirtschaftlich } \\
\text { erreicht }\end{array}$ & 310 & 62,2 & 5 & 1,6 & 11 & 3,5 & 27 & 8,7 & 67 & 21,6 & 17 & 5,5 & 74 & 23,9 & 20 & 6,5 & 24 & 7,7 & 65 & 21,0 \\
\hline Förderungsbeiträge *) & 46,2 & 66,5 & 0,2 & 0,4 & 1,8 & 4,0 & 2,7 & 5,9 & 11,5 & 24,9 & 2,2 & 4,7 & 14,2 & 30,8 & 2,5 & 5,4 & 3,0 & 6,4 & 8,1 & 17,5 \\
\hline Darlehen *) & 32,9 & 65,5 & 0,6 & 2,0 & 1,5 & 4,7 & 2,0 & 6,1 & 6,4 & 19,3 & 2,1 & 6,5 & 9,3 & 28,1 & 2,6 & 7,8 & 3,0 & 9,1 & 5,4 & 16,3 \\
\hline
\end{tabular}

*) Beträge in Millionen Euro 
Tabelle 22 Frage 8: Wurde das Projektziel aus technisch/wirtschaftlicher Sicht erreicht? Nach Beschäftigtengrößenklassen

\begin{tabular}{|c|c|c|c|c|c|c|c|c|c|c|c|c|c|c|c|c|}
\hline & \multicolumn{2}{|c|}{ Insgesamt } & \multicolumn{2}{|c|}{ von 1 bis 20} & \multicolumn{2}{|c|}{ von 21 bis 50} & \multicolumn{2}{|c|}{ von 51 bis 100} & \multicolumn{2}{|c|}{ von 101 bis 250} & \multicolumn{2}{|c|}{ von 251 bis 500} & \multicolumn{2}{|c|}{ von 501 bis 1000} & \multicolumn{2}{|c|}{ über 1000} \\
\hline & abs. & in $\%$ & abs. & in $\%$ & abs. & in $\%$ & abs. & in $\%$ & abs. & in $\%$ & abs. & in $\%$ & abs. & in $\%$ & abs. & in $\%$ \\
\hline Projekte insgesamt & 468 & 100,0 & 123 & 26,3 & 58 & 12,4 & 50 & 10,7 & 77 & 16,5 & 46 & 9,8 & 45 & 9,6 & 69 & 14,7 \\
\hline Förderungsbeiträge *) & 66,8 & 100,0 & 8,3 & 12,4 & 5,7 & 8,5 & 7,3 & 10,9 & 12,0 & 17,9 & 7,2 & 10,8 & 9,4 & 14,0 & 17,0 & 25,4 \\
\hline Darlehen *) & 47,3 & 100,0 & 6,3 & 13,2 & 4,3 & 9,1 & 7,3 & 15,4 & 8,0 & 17,0 & 5,6 & 11,9 & 8,3 & 17,6 & 7,5 & 15,8 \\
\hline technischer Sicht & 417 & 89,1 & 112 & 26,9 & 52 & 12,5 & 43 & 10,3 & 68 & 16,3 & 40 & 9,6 & 40 & 9,6 & 62 & 14,9 \\
\hline Förderungsbeiträge *) & 60,4 & 90,4 & 7,3 & 12,1 & 5,0 & 8,3 & 6,6 & 10,9 & 10,8 & 17,9 & 6,8 & 11,2 & 8,4 & 13,8 & 15,6 & 25,9 \\
\hline Darlehen *) & 42,7 & 90,2 & 5,6 & 13,1 & 3,6 & 8,4 & 7,0 & 16,4 & 7,1 & 16,7 & 5,0 & 11,8 & 8,1 & 19,0 & 6,2 & 14,6 \\
\hline wirtschaftlicher Sicht & 294 & 62,8 & 70 & 23,8 & 39 & 13,3 & 29 & 9,9 & 54 & 18,4 & 29 & 9,9 & 26 & 8,8 & 47 & 16,0 \\
\hline Förderungsbeiträge *) & 44,7 & 66,8 & 4,3 & 9,6 & 3,8 & 8,6 & 5,3 & 11,9 & 8,8 & 19,7 & 5,5 & 12,3 & 4,5 & 10,1 & 12,4 & 27,8 \\
\hline Darlehen *) & 30,9 & 65,4 & 2,9 & 9,5 & 2,9 & 9,5 & 6,1 & 19,7 & 5,6 & 17,9 & 3,3 & 10,5 & 5,2 & 16,9 & 4,9 & 15,9 \\
\hline
\end{tabular}

*) Beträge in Millionen Euro 
Tabelle 23 Frage 8: Wurde das Projektziel aus technisch/wirtschaftlicher Sicht erreicht? Nach ÖNACE Klassifikation der Projekte

\begin{tabular}{|c|c|c|c|c|c|c|c|c|c|c|c|c|c|c|c|c|c|c|}
\hline & \multicolumn{2}{|c|}{ Insgesamt } & \multicolumn{2}{|c|}{ Sektor A, B } & \multicolumn{2}{|c|}{ Sektor C } & \multicolumn{2}{|c|}{ Sektor D, E, F } & \multicolumn{2}{|c|}{ Sektor G } & \multicolumn{2}{|c|}{ Sektor J } & \multicolumn{2}{|c|}{ Sektor K, L, N } & \multicolumn{2}{|c|}{ Sektor M } & \multicolumn{2}{|c|}{ Sonstige } \\
\hline & abs. & in $\%$ & abs. & in $\%$ & abs. & in $\%$ & abs. & in $\%$ & abs. & in $\%$ & abs. & in $\%$ & abs. & in $\%$ & abs. & in $\%$ & abs. & in $\%$ \\
\hline Projekte insgesamt & 498 & $100 \%$ & 5 & 1,0 & 284 & 57,0 & 7 & 1,4 & 34 & 6,8 & 49 & 9,8 & 11 & 2,2 & 59 & 11,8 & 49 & 9,8 \\
\hline Förderungsbeiträge *) & 69,4 & $100 \%$ & 0,3 & 0,4 & 48,2 & 69,4 & 0,4 & 0,6 & 5,8 & 8,4 & 4,6 & 6,6 & 1,3 & 1,9 & 4,7 & 6,8 & 4,14 & 6,0 \\
\hline Darlehen *) & 50,3 & $100 \%$ & 0,2 & 0,4 & 38,8 & 77,0 & 0,4 & 0,9 & 2,1 & 4,3 & 3,3 & 6,5 & 0,1 & 0,3 & 3,5 & 6,9 & 1,85 & 3,7 \\
\hline technischer Sicht & 441 & 88,6 & 4 & 0,9 & 253 & 57,4 & 4 & 0,9 & 31 & 7,0 & 47 & 10,7 & 10 & 2,3 & 52 & 11,8 & 40 & 9,1 \\
\hline Förderungsbeiträge *) & 62,6 & 90,2 & 0,2 & 0,3 & 44,5 & 71,1 & 0,3 & 0,5 & 5,7 & 9,1 & 4,4 & 7,1 & 0,6 & 1,0 & 3,6 & 5,8 & 3,3 & 5,2 \\
\hline Darlehen *) & 45,3 & 90,0 & 0,2 & 0,4 & 35,5 & 78,4 & 0,3 & 0,7 & 2,1 & 4,7 & 3,1 & 6,8 & 0,1 & 0,3 & 2,6 & 5,7 & 1,4 & 3,1 \\
\hline wirtschaftlicher Sicht & 310 & 62,2 & 2 & 0,6 & 179 & 57,7 & 5 & 1,6 & 23 & 7,4 & 34 & 11,0 & 7 & 2,3 & 33 & 10,6 & 27 & 8,7 \\
\hline Förderungsbeiträge *) & 46,2 & 66,5 & 0,1 & 0,2 & 32,0 & 69,4 & 0,3 & 0,7 & 5,0 & 10,8 & 3,7 & 7,9 & 0,3 & 0,7 & 2,2 & 4,7 & 2,6 & 5,6 \\
\hline Darlehen *) & 32,9 & 65,5 & 0,1 & 0,4 & 25,8 & 78,5 & 0,4 & 1,1 & 1,6 & 4,8 & 2,5 & 7,6 & 0,1 & 0,3 & 1,6 & 4,7 & 0,9 & 2,6 \\
\hline
\end{tabular}

*) Beträge in Millionen Euro

$\begin{array}{ll}\text { A: Land- und Forstwirtschaft; B: Bergbau } & \text { D:Abfallentsorgung/Beseitigung } \\ & \text { E: Umweltverschmutzung; F: Bau }\end{array}$

C: Herstellung von Waren
G: Handel, Instandhaltung und Reparatur von KFZ
$\mathrm{J}$ : Information und Kommunikation

K: Finanzdienstleistungen, L: Wohnungswesen $\mathrm{N}$ : Wirtschaftliche Dienstleistungen
M: Wissenschaftliche und technische Dienstleistungen

Sonstige, nicht eindeutig zuordenbare Projekte 
Tabelle 24 Frage 11: Werden die Projektergebnisse im Unternehmen wirtschaftlich verwertet? Im Bundesländervergleich

\begin{tabular}{|c|c|c|c|c|c|c|c|c|c|c|c|c|c|c|c|c|c|c|c|c|}
\hline & \multicolumn{2}{|c|}{ Insgesamt } & \multicolumn{2}{|c|}{ Burgenland } & \multicolumn{2}{|c|}{ Kärnten } & \multicolumn{2}{|c|}{$\begin{array}{l}\text { Niederöster- } \\
\text { reich }\end{array}$} & \multicolumn{2}{|c|}{$\begin{array}{l}\text { Oberöster- } \\
\text { reich }\end{array}$} & \multicolumn{2}{|c|}{ Salzburg } & \multicolumn{2}{|c|}{ Steiermark } & \multicolumn{2}{|c|}{ Tirol } & \multicolumn{2}{|c|}{ Vorarlberg } & \multicolumn{2}{|c|}{ Wien } \\
\hline & abs. & in $\%$ & abs. & in $\%$ & abs. & in $\%$ & abs. & in $\%$ & abs. & in $\%$ & abs. & in $\%$ & abs. & in $\%$ & abs. & in $\%$ & abs. & in $\%$ & abs. & in $\%$ \\
\hline Projekte insgesamt & 498 & 100 & 8 & 1,6 & 27 & 5,4 & 52 & 10,4 & 112 & 22,5 & 26 & 5,2 & 115 & 23,1 & 34 & 6,8 & 32 & 6,4 & 92 & 18,5 \\
\hline Förderungsbeiträge *) & 69,4 & 100,0 & 1,1 & 1,6 & 3,2 & 4,6 & 4,9 & 7,1 & 16,4 & 23,6 & 3,8 & 5,4 & 21,1 & 30,4 & 5,2 & 7,5 & 3,8 & 5,5 & 10,0 & 14,4 \\
\hline Darlehen *) & 50,3 & 100,0 & 0,9 & 1,8 & 3,0 & 6,0 & 4,1 & 8,2 & 10,3 & 20,4 & 3,1 & 6,2 & 14,1 & 28,0 & 4,1 & 8,1 & 4,1 & 8,1 & 6,7 & 13,3 \\
\hline $\begin{array}{l}\text { werden im Unternehmen } \\
\text { bereits wirtschaftlich } \\
\text { verwertet }\end{array}$ & 295 & 59,2 & 4 & 1,4 & 15 & 5,1 & 33 & 11,2 & 65 & 22,0 & 16 & 5,4 & 69 & 23,4 & 15 & 5,1 & 24 & 8,1 & 54 & 18,3 \\
\hline Förderungsbeiträge *) & 45,1 & 65,1 & 0,2 & 0,4 & 2,1 & 4,7 & 3,2 & 7,0 & 12,3 & 27,3 & 2,6 & 5,8 & 13,4 & 29,8 & 1,7 & 3,7 & 2,7 & 6,1 & 6,9 & 15,2 \\
\hline Darlehen *) & 30,8 & 61,2 & 0,6 & 2,1 & 2,1 & 7,0 & 2,9 & 9,3 & 5,5 & 18,0 & 1,9 & 6,2 & 8,0 & 26,1 & 1,6 & 5,1 & 3,2 & 10,4 & 4,8 & 15,8 \\
\hline $\begin{array}{l}\text { werden in Zukunft im } \\
\text { Unternehmen wirtschaft- } \\
\text { lich verwertet werden } \\
\text { können }\end{array}$ & 45 & 9,0 & 1 & 2,2 & 3 & 6,7 & 3 & 6,7 & 15 & 33,3 & 2 & 4,4 & 8 & 17,8 & 6 & 13,3 & 2 & 4,4 & 5 & 11,1 \\
\hline Förderungsbeiträge *) & 8,4 & 12,2 & 0,7 & 8,1 & 0,5 & 5,8 & 0,5 & 6,1 & 1,6 & 19,3 & 0,2 & 2,4 & 2,5 & 29,6 & 1,8 & 20,9 & 0,1 & 1,1 & 0,6 & 6,7 \\
\hline Darlehen *) & 6,8 & 13,5 & 0,0 & 0,0 & 0,2 & 3,5 & 0,5 & 6,6 & 1,2 & 18,3 & 0,3 & 4,4 & 2,1 & 30,4 & 1,8 & 26,1 & 0,1 & 1,3 & 0,6 & 9,2 \\
\hline $\begin{array}{l}\text { Im Unternehmen nicht } \\
\text { wirtschaftlich verwertbar, } \\
\text { aber sinnvoll für Erkennt- } \\
\text { niserweiterung }\end{array}$ & 114 & 22,9 & 3 & 2,6 & 6 & 5,3 & 12 & 10,5 & 19 & 16,7 & 6 & 5,3 & 28 & 24,6 & 9 & 7,9 & 5 & 4,4 & 26 & 22,8 \\
\hline Förderungsbeiträge *) & 10,3 & 14,9 & 0,2 & 2,0 & 0,4 & 4,0 & 0,7 & 7,0 & 1,6 & 15,0 & 0,7 & 6,4 & 3,2 & 30,6 & 0,6 & 5,4 & 0,9 & 8,6 & 2,2 & 21,0 \\
\hline Darlehen *) & 9,3 & 18,5 & 0,2 & 2,7 & 0,4 & 4,6 & 0,3 & 3,1 & 2,0 & 21,1 & 0,5 & 5,2 & 3,8 & 40,7 & 0,4 & 4,8 & 0,7 & 7,1 & 1,0 & 10,6 \\
\hline $\begin{array}{l}\text { können im Unternehmen } \\
\text { nicht verwertet werden }\end{array}$ & 25 & 5,0 & 0 & 0,0 & 1 & 4,0 & 2 & 8,0 & 6 & 24,0 & 1 & 4,0 & 8 & 32,0 & 3 & 12,0 & 1 & 4,0 & 3 & 12,0 \\
\hline Förderungsbeiträge *) & 7,9 & 11,4 & 0,0 & 0,0 & 0,1 & 1,0 & 0,1 & 1,7 & 0,4 & 4,9 & 0,1 & 1,7 & 1,8 & 23,2 & 1,2 & 14,7 & 0,1 & 1,6 & 4,1 & 51,2 \\
\hline Darlehen *) & 1,6 & 3,2 & 0,0 & 0,0 & 0,1 & 8,9 & 0,1 & 5,1 & 0,7 & 43,3 & 0,2 & 14,0 & 0,1 & 8,5 & 0,2 & 11,8 & 0,1 & 8,4 & 0,0 & 0,0 \\
\hline
\end{tabular}

*) Beträge in Millionen Euro 
Tabelle 25 Frage 11: Werden die Projektergebnisse im Unternehmen wirtschaftlich verwertet? Nach Beschäftigtengrößenklassen

\begin{tabular}{|c|c|c|c|c|c|c|c|c|c|c|c|c|c|c|c|c|}
\hline & \multicolumn{2}{|c|}{ Insgesamt } & \multicolumn{2}{|c|}{ von 1 bis 20} & \multicolumn{2}{|c|}{ von 21 bis 50} & \multicolumn{2}{|c|}{ von 51 bis 100} & \multicolumn{2}{|c|}{ von 101 bis 250} & \multicolumn{2}{|c|}{ von 251 bis 500} & \multicolumn{2}{|c|}{ von 501 bis 1000} & \multicolumn{2}{|c|}{ über 1000} \\
\hline & abs. & in $\%$ & abs. & in $\%$ & abs. & in $\%$ & abs. & in $\%$ & abs. & in $\%$ & abs. & in $\%$ & abs. & in $\%$ & abs. & in $\%$ \\
\hline Projekte insgesamt & 468 & 100,0 & 123 & 26,3 & 58 & 12,4 & 50 & 10,7 & 77 & 16,5 & 46 & 9,8 & 45 & 9,6 & 69 & 14,7 \\
\hline Gesamtförderung *) & 66,8 & 100,0 & 8,3 & 12,4 & 5,7 & 8,5 & 7,3 & 10,9 & 12,0 & 17,9 & 7,2 & 10,8 & 9,4 & 14,0 & 17,0 & 25,4 \\
\hline Darlehen *) & 47,3 & 100,0 & 6,3 & 13,2 & 4,3 & 9,1 & 7,3 & 15,4 & 8,0 & 17,0 & 5,6 & 11,9 & 8,3 & 17,6 & 7,5 & 15,8 \\
\hline $\begin{array}{l}\text { werden im Unternehmen bereits } \\
\text { wirtschaftlich verwertet }\end{array}$ & 284 & 60,7 & 64 & 22,5 & 28 & 9,9 & 35 & 12,3 & 56 & 19,7 & 26 & 9,2 & 31 & 10,9 & 44 & 15,5 \\
\hline Gesamtförderung *) & 44,1 & 65,9 & 4,2 & 9,5 & 3,1 & 6,9 & 4,3 & 9,7 & 9,5 & 21,7 & 5,2 & 11,9 & 6,7 & 15,2 & 11,0 & 25,1 \\
\hline Darlehen *) & 29,2 & 61,8 & 3,7 & 12,7 & 2,2 & 7,4 & 3,5 & 12,0 & 6,4 & 21,8 & 3,2 & 10,9 & 6,2 & 21,1 & 4,1 & 14,1 \\
\hline $\begin{array}{l}\text { werden in Zukunft im Unternehmen } \\
\text { wirtschaftlich verwertet werden } \\
\text { können }\end{array}$ & 40 & 8,5 & 9 & 22,5 & 9 & 22,5 & 5 & 12,5 & 0 & 0,0 & 4 & 10,0 & 7 & 17,5 & 6 & 15,0 \\
\hline Gesamtförderung *) & 7,8 & 11,6 & 0,6 & 8,0 & 1,1 & 14,7 & 0,7 & 9,5 & 0,0 & 0,0 & 0,2 & 3,1 & 2,2 & 28,5 & 2,8 & 36,1 \\
\hline Darlehen *) & 6,0 & 12,6 & 0,8 & 12,6 & 0,9 & 15,0 & 0,3 & 5,5 & 0,0 & 0,0 & 0,3 & 5,6 & 1,9 & 31,1 & 1,8 & 30,2 \\
\hline $\begin{array}{l}\text { Im Unternehmen nicht wirtschaftlich } \\
\text { verwertbar, aber sinnvoll für Er- } \\
\text { kenntniserweiterung }\end{array}$ & 104 & 22,2 & 43 & 41,3 & 13 & 12,5 & 7 & 6,7 & 12 & 11,5 & 11 & 10,6 & 6 & 5,8 & 12 & 11,5 \\
\hline Gesamtförderung *) & 9,7 & 14,5 & 3,1 & 32,0 & 0,9 & 9,6 & 2,2 & 22,3 & 1,1 & 11,5 & 1,2 & 12,1 & 0,4 & 4,0 & 0,8 & 8,5 \\
\hline Darlehen *) & 8,7 & 18,4 & 1,4 & 16,3 & 0,8 & 9,5 & 3,3 & 38,0 & 0,7 & 7,5 & 1,3 & 14,8 & 0,2 & 2,2 & 1,0 & 11,7 \\
\hline $\begin{array}{l}\text { können im Unternehmen nicht } \\
\text { verwertet werden }\end{array}$ & 22 & 4,7 & 4 & 18,2 & 2 & 9,1 & 3 & 13,6 & 5 & 22,7 & 2 & 9,1 & 0 & 0,0 & 6 & 27,3 \\
\hline Gesamtförderung *) & 3,9 & 5,8 & 0,3 & 7,4 & 0,1 & 2,2 & 0,1 & 2,5 & 1,0 & 25,5 & 0,2 & 4,7 & 0,0 & 0,0 & 2,2 & 57,7 \\
\hline Darlehen *) & 1,5 & 3,1 & 0,3 & 22,2 & 0,0 & 2,9 & 0,1 & 8,7 & 0,5 & 30,8 & 0,2 & 13,7 & 0,0 & 0,0 & 0,3 & 21,6 \\
\hline
\end{tabular}

*) Beträge in Millionen Euro 
Tabelle 26 Frage 11: Werden die Projektergebnisse im Unternehmen wirtschaftlich verwertet? Nach ÖNACE Klassifikation der Projekte

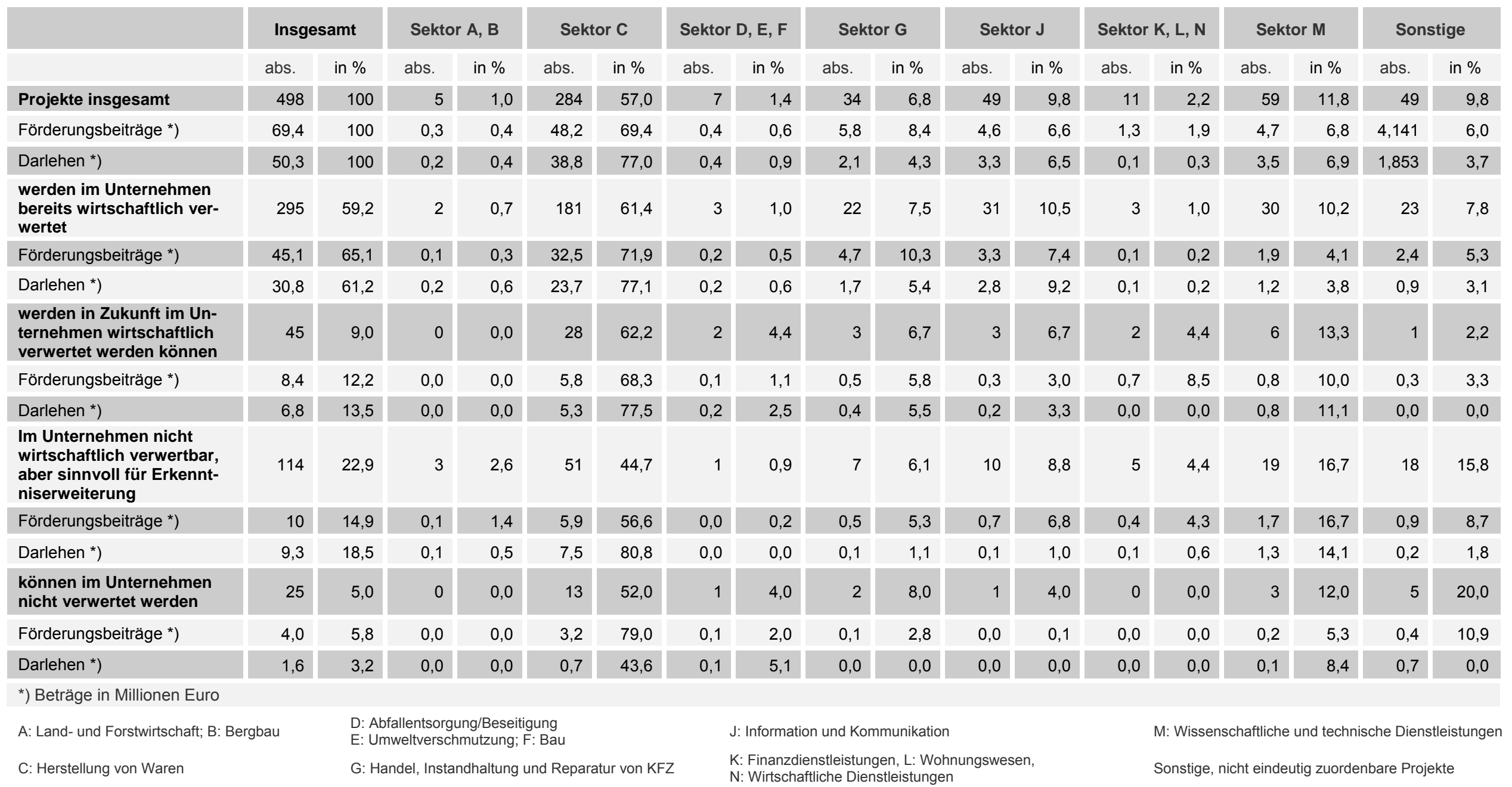


Tabelle 27 Kooperationsverhalten im Bundesländervergleich

Frage 23: Sind neue Kontakte/Kooperationen entstanden?

\begin{tabular}{|c|c|c|c|c|c|c|c|c|c|c|c|c|c|c|c|c|c|c|c|c|}
\hline & \multicolumn{2}{|c|}{ Insgesamt } & \multicolumn{2}{|c|}{ Burgenland } & \multicolumn{2}{|c|}{ Kärnten } & \multicolumn{2}{|c|}{$\begin{array}{l}\text { Niederöster- } \\
\text { reich }\end{array}$} & \multicolumn{2}{|c|}{$\begin{array}{l}\text { Oberöster- } \\
\text { reich }\end{array}$} & \multicolumn{2}{|c|}{ Salzburg } & \multicolumn{2}{|c|}{ Steiermark } & \multicolumn{2}{|c|}{ Tirol } & \multicolumn{2}{|c|}{ Vorarlberg } & \multicolumn{2}{|c|}{ Wien } \\
\hline & abs. & in $\%$ & abs. & in $\%$ & abs. & in $\%$ & abs. & in $\%$ & abs. & in $\%$ & abs. & in $\%$ & abs. & in $\%$ & abs. & in $\%$ & abs. & in $\%$ & abs. & in $\%$ \\
\hline $\begin{array}{l}\text { Neue Kontakte } \\
\text { entstanden *) }\end{array}$ & 292 & 59,3 & 4 & 50,0 & 16 & 59,3 & 29 & 55,8 & 70 & 62,5 & 22 & 84,6 & 63 & 54,8 & 16 & 47,1 & 19 & 59,4 & 53 & 57,6 \\
\hline $\begin{array}{l}\text { bestehende Kontak- } \\
\text { te intensiviert }{ }^{*} \text { ) }\end{array}$ & 317 & 64,4 & 4 & 50,0 & 14 & 51,9 & 33 & 63,5 & 70 & 62,5 & 19 & 73,1 & 85 & 73,9 & 21 & 61,8 & 20 & 62,5 & 51 & 55,4 \\
\hline keines von beiden *) & 64 & 13,0 & 2 & 25,0 & 5 & 18,5 & 5 & 9,6 & 13 & 11,6 & 1 & 3,8 & 10 & 8,7 & 7 & 20,6 & 3 & 9,4 & 18 & 19,6 \\
\hline weiß nicht *) & 15 & 3,0 & 0 & 0,0 & 1 & 3,7 & 2 & 3,8 & 1 & 0,9 & 1 & 3,8 & 5 & 4,3 & 0 & 0,0 & 2 & 6,3 & 3 & 3,3 \\
\hline
\end{tabular}

Frage 24: Wurden Kontakte nach Projektabschluss weitergeführt?

\begin{tabular}{|c|c|c|c|c|c|c|c|c|c|c|c|c|c|c|c|c|c|c|c|c|}
\hline & \multicolumn{2}{|c|}{ Insgesamt } & \multicolumn{2}{|c|}{ Burgenland } & \multicolumn{2}{|c|}{ Kärnten } & \multicolumn{2}{|c|}{$\begin{array}{l}\text { Niederöster- } \\
\text { reich }\end{array}$} & \multicolumn{2}{|c|}{$\begin{array}{l}\text { Oberöster- } \\
\text { reich }\end{array}$} & \multicolumn{2}{|c|}{ Salzburg } & \multicolumn{2}{|c|}{ Steiermark } & \multicolumn{2}{|c|}{ Tirol } & \multicolumn{2}{|c|}{ Vorarlberg } & \multicolumn{2}{|c|}{ Wien } \\
\hline & abs. & in $\%$ & abs. & in $\%$ & abs. & in $\%$ & abs. & in $\%$ & abs. & in $\%$ & abs. & in $\%$ & abs. & in $\%$ & abs. & in $\%$ & abs. & in $\%$ & abs. & in $\%$ \\
\hline $\begin{array}{l}\text { In Folgeprojekten } \\
\text { weitergeführt *) }\end{array}$ & 271 & 64,5 & 4 & 50,0 & 9 & 33,3 & 25 & 48,1 & 65 & 58,0 & 16 & 61,5 & 70 & 60,9 & 13 & 38,2 & 16 & 50,0 & 53 & 57,6 \\
\hline $\begin{array}{l}\text { in Publikationen } \\
\text { weitergeführt *) }\end{array}$ & 53 & 12,6 & 0 & 0,0 & 2 & 7,4 & 2 & 3,8 & 7 & 6,3 & 4 & 15,4 & 18 & 15,7 & 4 & 11,8 & 0 & 0,0 & 16 & 17,4 \\
\hline $\begin{array}{l}\text { ohne konkrete } \\
\text { Projekte weiterge- } \\
\text { führt *) }\end{array}$ & 135 & 32,1 & 2 & 25,0 & 10 & 37,0 & 22 & 42,3 & 34 & 30,4 & 9 & 34,6 & 27 & 23,5 & 7 & 20,6 & 8 & 25,0 & 16 & 17,4 \\
\hline nicht weitergeführtt*) & 42 & 10,0 & 0 & 0,0 & 3 & 11,1 & 3 & 5,8 & 10 & 8,9 & 2 & 7,7 & 11 & 9,6 & 5 & 14,7 & 4 & 12,5 & 4 & 4,3 \\
\hline
\end{tabular}


Tabelle 28 Kooperationsverhalten nach Beschäftigtengrößenklassen

Frage 23: Sind neue Kontakte/Kooperationen entstanden?

\begin{tabular}{|c|c|c|c|c|c|c|c|c|c|c|c|c|c|c|c|c|}
\hline & \multicolumn{2}{|c|}{ Insgesamt } & \multicolumn{2}{|c|}{ von 1 bis 20} & \multicolumn{2}{|c|}{ von 21 bis 50} & \multicolumn{2}{|c|}{ von 51 bis 100} & \multicolumn{2}{|c|}{ von 101 bis 250} & \multicolumn{2}{|c|}{ von 251 bis 500} & \multicolumn{2}{|c|}{ von 501 bis 1000} & \multicolumn{2}{|c|}{ über 1000} \\
\hline & absolut & in $\%$ & absolut & in $\%$ & absolut & in $\%$ & absolut & in $\%$ & absolut & in $\%$ & absolut & in $\%$ & absolut & in $\%$ & absolut & in $\%$ \\
\hline $\begin{array}{l}\text { Neue Kontakte ent- } \\
\text { standen *) }\end{array}$ & 276 & 59,0 & 74 & 60,2 & 35 & 60,3 & 31 & 62,0 & 43 & 55,8 & 28 & 60,9 & 27 & 60,0 & 38 & 55,1 \\
\hline $\begin{array}{l}\text { bestehende Kontakte } \\
\text { intensiviert *) }\end{array}$ & 297 & 63,5 & 71 & 57,7 & 33 & 56,9 & 28 & 56,0 & 53 & 68,8 & 32 & 69,6 & 31 & 68,9 & 49 & 71,0 \\
\hline keines von beiden *) & 61 & 13,0 & 17 & 13,8 & 11 & 19,0 & 7 & 14,0 & 8 & 10,4 & 3 & 6,5 & 8 & 17,8 & 7 & 10,1 \\
\hline weiß nicht *) & 13 & 2,8 & 1 & 0,8 & 2 & 3,4 & 2 & 4,0 & 4 & 5,2 & 2 & 4,3 & 0 & 0,0 & 2 & 2,9 \\
\hline
\end{tabular}

Frage 24: Wurden Kontakte nach Projektabschluss weitergeführt?

\begin{tabular}{|c|c|c|c|c|c|c|c|c|c|c|c|c|c|c|c|c|}
\hline & \multicolumn{2}{|c|}{ Insgesamt } & \multicolumn{2}{|c|}{ von 1 bis 20} & \multicolumn{2}{|c|}{ von 21 bis 50} & \multicolumn{2}{|c|}{ von 51 bis 100} & \multicolumn{2}{|c|}{ von 101 bis 250} & \multicolumn{2}{|c|}{ von 251 bis 500} & \multicolumn{2}{|c|}{ von 501 bis 1000} & \multicolumn{2}{|c|}{ über 1000} \\
\hline & absolut & in $\%$ & absolut & in $\%$ & absolut & in $\%$ & absolut & in $\%$ & absolut & in $\%$ & absolut & in $\%$ & absolut & in $\%$ & absolut & in $\%$ \\
\hline $\begin{array}{l}\text { In Folgeprojekten } \\
\text { weitergeführt *) }\end{array}$ & 257 & 65,1 & 64 & 52,0 & 28 & 48,3 & 28 & 56,0 & 45 & 58,4 & 28 & 60,9 & 26 & 57,8 & 38 & 55,1 \\
\hline $\begin{array}{l}\text { in Publikationen } \\
\text { weitergeführt *) }\end{array}$ & 51 & 12,9 & 12 & 9,8 & 6 & 10,3 & 6 & 12,0 & 6 & 7,8 & 8 & 17,4 & 5 & 11,1 & 8 & 11,6 \\
\hline $\begin{array}{l}\text { ohne konkrete Projek- } \\
\text { te weitergeführt *) }\end{array}$ & 127 & 32,2 & 37 & 30,1 & 20 & 34,5 & 11 & 22,0 & 19 & 24,7 & 11 & 23,9 & 12 & 26,7 & 17 & 24,6 \\
\hline nicht weitergeführt *) & 38 & 9,6 & 10 & 8,1 & 2 & 3,4 & 2 & 4,0 & 6 & 7,8 & 5 & 10,9 & 3 & 6,7 & 10 & 14,5 \\
\hline
\end{tabular}


Tabelle 29 Kooperationsverhalten nach ÖNACE Klassifikation der Projekte

Frage 23: Sind neue Kontakte/Kooperationen entstanden?

\begin{tabular}{|c|c|c|c|c|c|c|c|c|c|c|c|c|c|c|c|c|c|c|}
\hline & \multicolumn{2}{|c|}{ insgesamt } & \multicolumn{2}{|c|}{ Sektor A,B } & \multicolumn{2}{|c|}{ Sektor C } & \multicolumn{2}{|c|}{ Sektor D,E,F } & \multicolumn{2}{|c|}{ Sektor G } & \multicolumn{2}{|c|}{ Sektor J } & \multicolumn{2}{|c|}{ Sektor K,L,N } & \multicolumn{2}{|c|}{ Sektor M } & \multicolumn{2}{|c|}{ Sonstige } \\
\hline & abs. & in $\%$ & abs. & in $\%$ & abs. & in $\%$ & abs. & in $\%$ & abs. & in $\%$ & abs. & in $\%$ & abs. & in $\%$ & abs. & in $\%$ & abs. & in $\%$ \\
\hline $\begin{array}{l}\text { Neue Kontakte ent- } \\
\text { standen *) }\end{array}$ & 291 & 59,1 & 2 & 40,0 & 167 & 7,3 & 4 & 57,1 & 14 & 41,2 & 30 & 61,2 & 7 & 63,6 & 36 & 61,0 & 31 & 63,3 \\
\hline $\begin{array}{l}\text { bestehende Kontakte } \\
\text { intensiviert *) }\end{array}$ & 316 & 64,2 & 5 & 100,0 & 191 & 8,4 & 4 & 57,1 & 22 & 64,7 & 29 & 59,2 & 4 & 36,4 & 30 & 50,8 & 31 & 63,3 \\
\hline keines von beiden *) & 65 & 13,2 & 0 & 0,0 & 29 & 1,3 & 2 & 28,6 & 7 & 20,6 & 10 & 20,4 & 3 & 27,3 & 7 & 11,9 & 7 & 14,3 \\
\hline weiß nicht *) & 15 & 3,0 & 0 & 0,0 & 9 & 0,4 & 0 & 0,0 & 1 & 2,9 & 2 & 4,1 & 1 & 9,1 & 1 & 1,7 & 1 & 2,0 \\
\hline
\end{tabular}

Frage 24: Wurden Kontakte nach Projektabschluss weitergeführt?

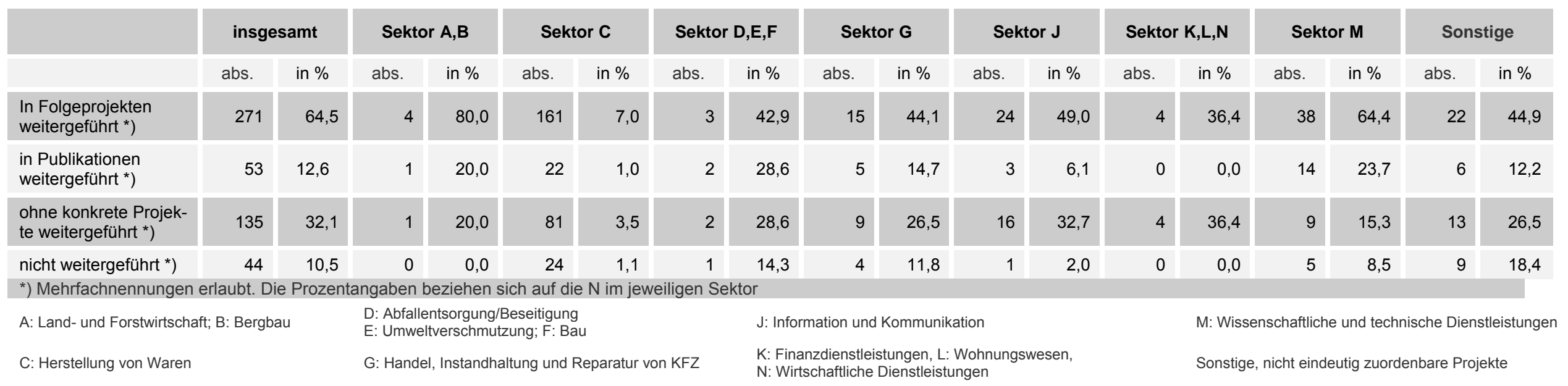


KMU Forschung Austria

Austrian Institute for SME Research 